\title{
Modeling Multi-Dimensional Public Opinion Process Based on Complex Network Dynamics Model in the Context of Derived Topics
}

\author{
Tinggui Chen ${ }^{1,2, *} \mathbb{C}$, Xiaohua Yin ${ }^{1}$, Jianjun Yang ${ }^{3}$, Guodong Cong ${ }^{4}$ and Guoping Li $^{5}$ \\ 1 School of Statistics and Mathematics, Zhejiang Gongshang University, Hangzhou 310018, China; \\ yinxh0213@163.com \\ 2 Academy of Zhejiang Culture Industry Innovation \& Development, Zhejiang Gongshang University, \\ Hangzhou 310018, China \\ 3 Department of Computer Science and Information Systems, University of North Georgia, \\ Oakwood, GA 30566, USA; Jianjun.Yang@ung.edu \\ 4 School of Tourism and Urban-Rural Planning, Zhejiang Gongshang University, Hangzhou 310018, China; \\ cgd@mail.zjgsu.edu.cn \\ 5 Zhejiang Liziyuan Food Co., Ltd., Hangzhou 310018, China; winnerwon@163.com \\ * Correspondence: ctgsimon@mail.zjgsu.edu.cn
}

Citation: Chen, T.; Yin, X.; Yang, J.; Cong, G.; Li, G. Modeling

Multi-Dimensional Public Opinion

Process Based on Complex Network Dynamics Model in the Context of Derived Topics. Axioms 2021, 10, 270. https://doi.org/10.3390/

axioms 10040270

Academic Editor: Palle E.T. Jorgensen

Received: 1 September 2021

Accepted: 19 October 2021

Published: 21 October 2021

Publisher's Note: MDPI stays neutral with regard to jurisdictional claims in published maps and institutional affiliations.

Copyright: (c) 2021 by the authors. Licensee MDPI, Basel, Switzerland. This article is an open access article distributed under the terms and conditions of the Creative Commons Attribution (CC BY) license (https:// creativecommons.org/licenses/by/ $4.0 /)$.

\begin{abstract}
With the rapid development of the Internet, the speed with which information can be updated and propagated has accelerated, resulting in wide variations in public opinion. Usually, after the occurrence of some newsworthy event, discussion topics are generated in networks that influence the formation of initial public opinion. After a period of propagation, some of these topics are further derived into new subtopics, which intertwine with the initial public opinion to form a multidimensional public opinion. This paper is concerned with the formation process of multidimensional public opinion in the context of derived topics. Firstly, the initial public opinion variation mechanism is introduced to reveal the formation process of derived subtopics, then Brownian motion is used to determine the subtopic propagation parameters and their propagation is studied based on complex network dynamics according to the principle of evolution. The formula of basic reproductive number is introduced to determine whether derived subtopics can form derived public opinion, thereby revealing the whole process of multi-dimensional public opinion formation. Secondly, through simulation experiments, the influences of various factors, such as the degree of information alienation, environmental forces, topic correlation coefficients, the amount of information contained in subtopics, and network topology on the formation of multi-dimensional public opinion are studied. The simulation results show that: (1) Environmental forces and the amount of information contained in subtopics are key factors affecting the formation of multi-dimensional public opinion. Among them, environmental forces have a greater impact on the number of subtopics, and the amount of information contained in subtopics determines whether the subtopic can be the key factor that forms the derived public opinion. (2) Only when the degree of information alienation reaches a certain level, will derived subtopics emerge. At the same time, the degree of information alienation has a greater impact on the number of derived subtopics, but it has a small impact on the dimensions of the final public opinion. (3) The network topology does not have much impact on the number of derived subtopics but has a greater impact on the number of individuals participating in the discussion of subtopics. The multidimensional public opinion dimension formed by the network topology with a high aggregation coefficient and small average path length is higher. Finally, a practical case verifies the rationality and effectiveness of the model proposed in this paper.
\end{abstract}

Keywords: multi-dimensional public opinion; topic derivation; complex network dynamics model; online comments; hot events 


\section{Introduction}

In the era of big data, massive amounts of information are generated on social platforms. Each netizen can make comments on a hot topic based on the information the individual has obtained. When the topic is sufficiently popular, an initial public opinion will form about the event in question. At the same time, with the further disclosure of information, related derived subtopics may be generated and more netizens may join the discussion. Along with the evolution of initial public opinion and the influence of various external factors, some derived subtopics will form a second stage of public opinion [1]. This new derived public opinion and the initial public opinion are intertwined to form a multi-dimensional public opinion. In real life, with the evolution of emergency events and the change of related information, more initial public opinion will generate derived public opinion. Taking the topic "The COVID-19 incident at the end of 2019", for instance, the online opinion derived from "Wuhan epidemic" to other derived topics, such as "Conspiracy theory of the epidemic origin", "Li Wenliang, the first man who discovered the epidemic", and "U.S. congressmen concealed the epidemic". The discussions that form this multi-dimensional public opinion make people nervous and scary and this has a significant impact on social harmony and stability. Based on this, analyzing the formation mechanism of derived public opinion, as well as the formation process of multi-dimensional public opinion, has important theoretical and practical significance for studying public opinion.

At present, there is relatively little research into the formation process of multidimensional public opinion in the context of topic derivation. The main research methods are qualitative analysis and quantitative modeling [2]. In terms of qualitative analysis, scholars mostly use specific cases to discuss the definition, potential harm, and common characteristics of network-derived public opinion. In terms of quantitative modeling, researchers try to discover the rules of public opinion derivation by establishing models to better study its evolution so as to effectively prevent possible public opinion crises. Among them, the SIR infectious disease model is often used to analyze network derivation effects. However, currently, only a single-dimensional initial public opinion is analyzed, and multidimensional public opinion is rarely considered. In fact, under the influences of multiple information, initial public opinion often derives public opinion in multiple dimensions. Based on this, after the initial topic is discussed and the initial public opinion is formed, this paper first introduces the initial public opinion derivation mechanism to reveal the formation process of derived subtopics, and then analyzes its evolution law based on the SIR disease model and maps the propagation process of multiple subtopics into multiple layers. Basic reproduction number is introduced to determine whether derived subtopics can form derived public opinion, thereby revealing the entire process of multi-dimensional public opinion formation.

The structure of this paper is organized as follows: Section 2 is literature review; Section 3 builds a model for the formation of multi-dimensional public opinion in a topicderived context; Section 4 uses simulation experiments to analyze the influences of some of the main factors on the formation of multi-dimensional public opinion; Section 5 uses actual cases to verify the model proposed in this paper; Section 6 contains the conclusions of the study and considers the prospects for future work.

\section{Literature Review}

This paper defines multi-dimensional public opinion as public opinion formed by combining initial public opinion and derived public opinion. When single or multiple derived public opinions are generated, they together go to constitute multi-dimensional public opinion. Derived public opinion is the basis of multi-dimensional public opinion and the main subject of current research. Therefore, the discussion in this paper mainly focuses on the formation of derived public opinion. At present, many scholars have conducted research on the formation of derived public opinion, which mainly includes two aspects: one is the study of the propagation and evolutionary mechanism of derived 
public opinion; the other is the study of the reasons for the formation of derived public opinion and countermeasures to it.

At present, the research on the propagation and evolutionary mechanism of derived public opinion is mostly based on infectious disease model, but the focus is on specific public opinion topics, such as the research on rumors and fake news events. The representative literatures are as follows: Lan et al. [3] set up a mathematic model of the derivative effect of network public opinion based on a logistic model from the viewpoint of information alienation. Zhang and Feng [4] put forward a two-layer coupled SEIR public opinion propagation model for derived topics that applied when news was circulated. Korobeinikov [5] studied global properties of SIR and SEIR epidemic models with multiple parallel infectious stages and verified that these systems possessed the only globally stable equilibrium state. Arenas et al. [6] adopted a Microscopic Markov Chain Approach (MMCA) meta population mobility model to study the cases of COVID-19. Estrada [7] modeled the Singapore COVID-19 pandemic with an SEIR multiplex network model. Yang et al. [8] proposed a competitive diffusion model, namely, the Linear Threshold model with One Direction state Transition (LT1DT), in order to explore the problem of minimizing the spread of rumor in social networks. Zanette and Damian [9] explored the dynamics of an epidemic-like model for the spread of a rumor on a small-world network. Moreno et al. [10] deduced the mean-field equations used to describe the dynamics of a rumor process occurring on top of complex heterogeneous networks. Zhou et al. [11] considered the influence of network topological structure and the unequal footings of neighbors of an infected node in propagating the rumor and found that the number of final infected nodes depended on the topology of the network. Tan et al. [12] organically combined the analytic hierarchy process and wavelet neural network to develop an effective and feasible network public opinion monitoring system, and analyzed the Theater High Altitude Area Defense (THAAD) incident as a case, verifying that the system had good evaluation performance and estimation accuracy. You et al. [13] proposed a social network-oriented public opinion monitoring platform based on ElasticSearch (SNES), and proved with a large body of empirical evidence that the platform could well adapt to social networks with high real-time data and good performance in public opinion monitoring. Chen et al. [14] proposed a monitoring and identification method for high-risk users of enterprise public opinion combined with user portrait technology and a random forest algorithm. The proposed scheme helped enterprises identify high-risk users with inadequate experience who may trigger negative public opinion. Wang et al. [15] proposed an improved energy model to characterize the propagation of rumors on social networks quantitatively and used experiments to evaluate the influences of model parameters, network structures, and effective linkage rates. Askarizadeh et al. [16] presented an evolutionary game model to analyze the rumor process in social networks and the analysis results showed that propagation of convincing anti-rumor messages and the location of rumor control centers had an important effect on debunking rumors. Although the aforementioned literature presents a propagation model of derived public opinion, it mostly uses topic derivation rate parameters to quantify the topic derivation process, and only explores the influence of the change of topic derivation rates on initial public opinion along a single dimension and does not consider multi-dimensional public opinion situations with the combination of derived public opinion and initial public opinion.

Some scholars have conducted research on the reasons for opinion formation and the response strategies of derived public opinion, and the representative literatures are as follows: From the viewpoint of spreading factors, Zhang [17] studied causes of network public opinion derivation triggered by public emergencies and concluded that the government, the media, and the public were important factors in the formation of derived public opinion, their different behavioral logics based on their respective interests in spreading information being the fundamental reason for the generation of derived public opinion. Duncan and Peter [18] found that large cascades of influence were driven not by influential individuals but by a critical mass of easily influenced individuals. Li [19] considered 
that the fundamental reason for the formation of derived network public opinion lies in information alienation. Snyder et al. [20] held that many large-scale phenomena, such as rapid changes in public opinion and the outbreak of disease epidemics, could be fruitfully modeled as cascades of activation on networks. In addition, according to the general law of network derived public opinion, Wang and Dai [21] summed up three basic derived chain structure types and corresponding probability algorithms, and finally listed the operational steps of a network derived public opinion chain. Moreover, by introducing social preference theory, Chen et al. [22] revealed the micro-interaction mechanism of public opinion polarization and their simulation results showed that different social preferences held by individuals had different influences on public opinion polarization effects. Although the above-mentioned literature reveals the formation and propagation mechanisms of derived public opinion to a certain extent, its research methods are mostly inductive, and are mostly based on single-dimensional network public opinion development. Few scholars adopt the perspective of multi-dimensional public opinion.

In addition, a number of scholars have conducted preliminary discussions of the multi-dimensional public opinion evolution model. By combining social judgment theory with the multi-agent model, $\mathrm{Li}$ and Xiao [23] proposed a multidimensional opinion evolution model for studying the dynamics of opinion polarization and the simulation results demonstrated that the polarization process was affected by assimilation effect parameters and contrast effect parameters. Parsegov et al. [24] proposed a significant extension of the classical Friedkin-Johnsen model so as to describe the evolution of agents' opinions on several topics. In addition, based on the real processes by which multiple topics concerning the same event were generated and disseminated, Sun and Chai [25] divided the portraits of online learners into three dimensions and constructed a labeling system for the portraits of learners based on the data fields of an online learning platform. Wang et al. [26] designed a topic detection algorithm that worked on these multidimensional public opinion networks and their simulation results demonstrated that this model could be used to effectively characterize the communication characteristics of multiple topics on "We the Media" networks. Although the above literature considers the multi-dimensional characteristics of online public opinion and reveals its evolution mechanism to a certain extent, most scholars directly discuss its nature and characteristics from the perspective of multi-dimensional public opinion. Few scholars focus on its formation mechanisms. In the propagation of subtopics, few scholars consider the interweaving and the mutual influence of multi-dimensional derived public opinion and initial public opinion. This has resulted in an incomplete understanding of the phenomena, and further research is needed.

In summary, current scholars mostly use single-dimensional online public opinion models to conduct research. Few scholars combine derived public opinion with initial public opinion and do not discuss the formation process of multi-dimensional public opinion from the perspective of derived topics. In reality, after the outbreak of an initial public opinion on a topic, multiple subtopics are often derived from the network. Some of these subtopics form derived public opinion in the propagation process and they are intertwined with the initial public opinion to form a more influential multi-dimensional public opinion. Based on this, from the perspective of derived topics, the formation mechanism of multi-dimensional public opinion can be studied more clearly. This paper discusses the formation process of multi-dimensional public opinion in the context of derived topics, in order to provide a reasonable guide.

\section{Model Construction}

The entire process of modeling multi-dimensional public opinion formation is based on complex network dynamics. First, the complex network simulation is used to generate the intricate relationship among netizens in the real world. In addition, the agent is used to represent the individual nodes in the network and the network scale is set to $N$, meaning it is assumed that there are $N$ nodes in the network, which are divided into three states: 
susceptible, infective, and recovered. At the same time, the SIR model is introduced to analyze the evolution of the three types.

Generally, for the initial topic formed by the emergent news worthy event, the perception of netizens and the propagation of events will prompt formation of an initial public opinion on the Internet. However, as time goes by, netizens' subjective understanding of the initial public opinion information will gradually become biased. With the gradual accumulation of deviations, the initial public opinion yield derivations, resulting in multiple subtopics. Some derived subtopics may form derived public opinions due to intense discussion and enthusiasm and a large amount of information. The intertwining of these with the earlier state of public opinion forms a multi-dimensional public opinion. Based on this, the specific research ideas of this paper are shown in Figure 1.

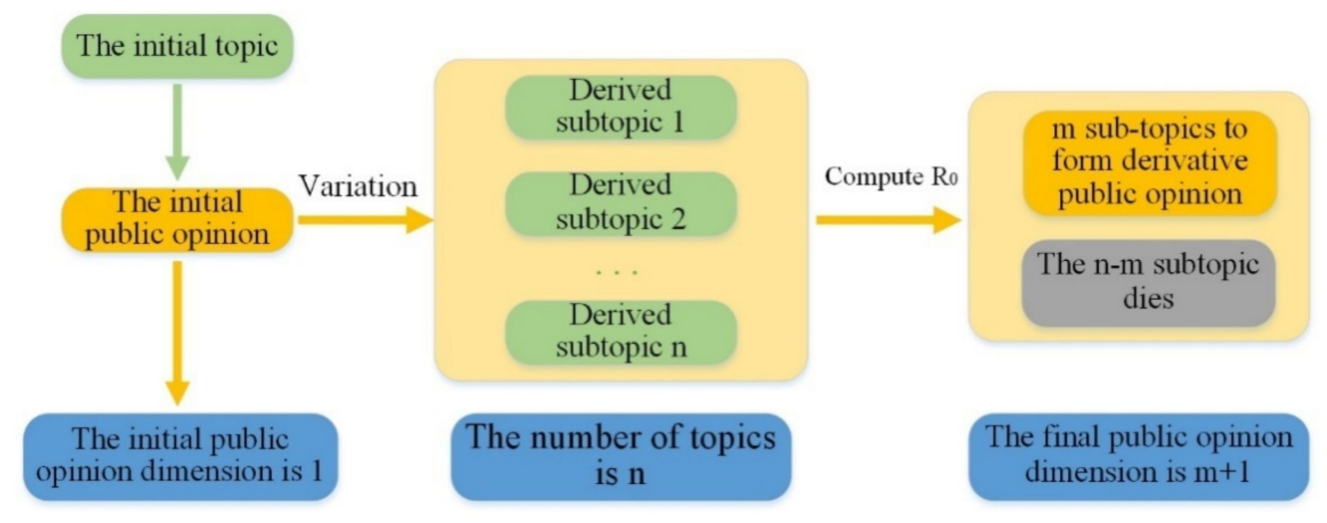

Figure 1. Research framework.

According to the Figure 1, this paper divides the formation process of multi-dimensional public opinion into four stages, as follows:

Stage 1: The formation and propagation stage of initial public opinion. For the initial topic formed by the emergent hot events, a large number of netizens participate in the discussion and spread the topic, prompting the initial topic to form an initial public opinion. The individual netizens participating in the initial public opinion discussion are divided into susceptible, infected and recovered status, and the SIR model is introduced to analyze their propagation process.

Stage 2: The generation stage of derived subtopics. As the initial public opinion spreads and the external environment is stimulated, the probability of the initial public opinion variation gradually increases. The variation degree $P$ is introduced to describe the variation degree of the initial public opinion. If the variation degree at time $T_{i}$ is at a high interval, it will generate a derived subtopic.

Stage 3: The information propagation stage of derived subtopics. After the derived subtopic is generated, netizens accept and pay attention to this source of information. When the individual's attention to the derived subtopic is greater than the threshold $g_{0}$ at time $T_{i}$, the individual will participate in the discussion of the sub-topic. Infection rate and immunity rate change based on Brownian motion. The subtopic information evolves according to the SIR model of the new propagation parameters.

Stage 4: The multi-dimensional public opinion formation stage. Although the initial public opinion may derive multiple subtopics, not all subtopics can form public opinion. Only when the propagation reproduction number $R_{0}$ of a derived subtopic reaches a certain threshold is it considered that the subtopic forms a derived public opinion, and the newly emerging derived public opinion is intertwined with the initial public opinion to form a multi-dimensional public opinion.

The parameters and variables involved in the formation of multi-dimensional public opinion are shown in Tables 1 and 2. 
Table 1. Relevant parameters.

\begin{tabular}{cc}
\hline Parameters & Description \\
\hline$\alpha$ & Infection rate \\
\hline$\beta$ & Immunity rate \\
\hline$\rho$ & Information alienation rate \\
\hline$\delta$ & Environmental forces \\
\hline$\theta_{i}$ & The topic correlation between the ith derived subtopic and the initial public opinion \\
\hline$\sigma_{i}$ & The amount of information contained in the $i$ th derived subtopic \\
\hline$P_{U}$ & The parameter of highly variable degree threshold \\
\hline$g_{0}$ & The attention threshold \\
\hline
\end{tabular}

Table 2. Relevant variables.

\begin{tabular}{cc}
\hline Variable & Description \\
\hline$S(t)$ & The numberof susceptible individuals in the network at time $t$ \\
\hline$I(t)$ & The number of infective individuals in the network at time $t$ \\
\hline$R(t)$ & The number of recovered individuals in the network at time $t$ \\
\hline$P$ & The degree of variation of initial public opinion \\
\hline$g_{i}$ & Individual $i$ 's attention to subtopics \\
\hline$R_{0}$ & Basic reproduction number \\
\hline
\end{tabular}

\subsection{Initial Public Opinion Propagation Model}

After the initial public opinion is formed, individuals in the social network begin to receive information and spread public opinion. The propagation process of initial public opinion is analyzed through the SIR model. First of all, individuals in the network are divided into three states in proportion, namely, susceptible state (S), infective state (I), and recovered state (R). $S$ is the class of netizens who have not received relevant public opinion information, I that of netizens who have received public opinion information and who actively spread it, $\mathrm{R}$ that of netizens who are not interested in public opinion information. The transformation relationship of the three is shown in Figure 2.

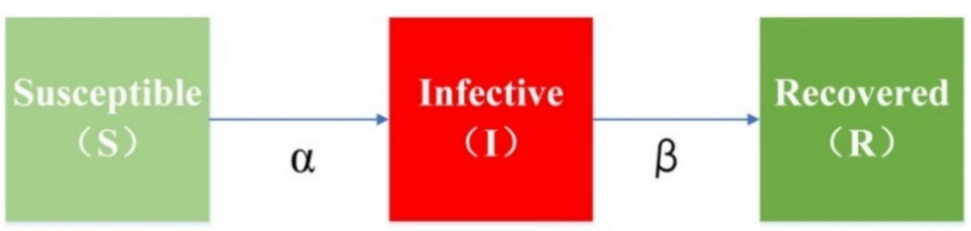

Figure 2. State transformation.

According to the Figure 2, when the public opinion information of a hot event is formed, susceptible individuals (S) will receive relevant information under the influence of surrounding infected individuals and thus become infective individuals (I). Assuming that there are $r$ susceptible individuals among the neighbors of the infective person at this time, the susceptible person will become infective with probability $\alpha$ after contact with the infective person, while infective individuals will gradually lose interest as time goes by and recover with probability $\beta$ [27].

Suppose that $S(t), I(t)$, and $R(t)$, respectively, represent the number of individuals in a susceptible state, an infective state, and a recovered state in the network at time $t$, and meet the following Formula (1):

$$
S(t)+I(t)+R(t)=N
$$


System dynamics equations are introduced to simulate the evolution of individuals in these three states in the network. Since dynamical equations are used only as a tool for public opinion evolution in this paper, the simple SIR model of rumor propagation in uniform network established by $\mathrm{Lu}$ [28] is used as the evolutionary model for initial public opinion. The dynamic equations are shown in the following Formula (2):

$$
\left\{\begin{array}{c}
\frac{d S(t)}{d t}=-\bar{k} * \alpha * I(t) * \frac{S(t)}{N} ; \\
\frac{d I(t)}{d t}=\bar{k} * \alpha * I(t) * \frac{S(t)}{N}-\beta * I(t) ; \\
\frac{d R(t)}{d t}=\beta * I(t) .
\end{array}\right.
$$

where $\bar{k}=\frac{1}{N} * \sum_{i}^{N} k(i)$ means the average degree of all nodes in the network. After the initial public opinion of the sudden hot event is formed, the infected node contacts with $\bar{k}$ neighbor nodes. Among them, the neighboring node in a susceptible state becomes infective with probability $\alpha$, and at the same time the infective node recovers with probability $\beta$.

\subsection{The Formation Process of Derived Subtopics}

Compared with traditional media, the spread of public opinion on the Internet is more sudden and unpredictable. After the initial public opinion of a newsworthy event is formed, related information will be viewed, commented on, and reposted by a large number of netizens in a relatively short period of time, its scope gradually expanding over time. With the continuous release of initial public opinion information, the netizens subjective understanding of initial public opinion will gradually generate deviations. With the gradual accumulation of these deviations, the information will be distorted in the process of propagation and then magnified by the influence of social network environments. In this process, the initial public opinion will change [29]. Generally speaking, the variation of public opinion events is multi-directional, and people's views on the same event tend to become diversified. Therefore, after public opinion becomes variable, multiple derived subtopics will usually be generated and the subtopics may be further derived to form a public opinion which is consistent with the initial public opinion and constitutes a multi-dimensional public opinion situation.

\subsubsection{The Degree of Variation $P$}

Generally, the process of public opinion variation is relatively slow, and the state of variation gradually deepens after a long period of accumulation. The degree of public opinion variation $P$ is introduced to reflect the state of initial public opinion variation. With the evolution of public opinion, $P$ slowly increases, and when $P$ reaches a certain threshold, the initial public opinion will be in a highly variable state, resulting in derived subtopics. As public opinion further develops and fades, $P$ also decreases to a smaller value. Generally speaking, the variation degree of public opinion is affected by many factors. The most important, however, is the degree of information alienation in the propagation of public opinion, along with the environmental forces of public opinion. The degree of information alienation $\rho$ refers to the contradiction between information produced and consumed and is due to the influence of various factors during the formation, propagation, and use of information, such that a subject loses the ability to handle incoming information, losing sight of the initial accounts, and becomes enslaved to and dominated by this new information [30]. For example, after the "Death of He Hongshen in Macao" event, due to changes in netizens' gossip psychology and attention perspective, the information reflecting the event itself lost its original significance and attention was displaced on to his family property, resulting in a series of subtopics such as "He's Original Wife" and "He's Property". Environmental force refers to the influence of the general environment, such as the propagation of events or network communication channels on public opinion, which is often affected by two aspects: firstly, there are the characteristics of the event itself, such as the degree of social disputes in different events; secondly, there is the impact of the network environment on events. $\delta$ is used to describe the strength of environmental 
forces. At present, with the prevalence of new media communication methods, information communication channels have become more diverse, and the network environment has played a guiding role in the opinions, attitudes and communication behaviors of netizens. The scope of the incident may gradually expand, and it is more likely to form public opinion. The incident has been distorted, leading to deviations from the original development trend, and given rise to derivations.

Combining the above two factors, according to the existing literature [31], the evolution process of public opinion variation is divided into a budding period, an outbreak period, a diffusion period, and a dissipative period. The variation process of public opinion is analyzed and the initial public opinion variation degree is described with the following Formula (3):

$$
P=\rho * e^{-\left(\frac{T-9}{\delta}\right)^{2}}
$$

where $T$ represents the evolution time of public opinion, $\rho$ is the degree of information alienation, $\delta$ is the environmental forces, and the degree of variation $P$ first increases and then decreases with the development of public opinion. Generally, a deeper degree of information alienation means a higher degree of variation, a greater environmental force, and a higher degree of variation.

\subsubsection{The Formation of Subtopics}

The initial public opinion variation degree is divided into two intervals, with $P_{U}$ as the threshold. When $0<P<P_{U}$, the degree of variation is lower; when $P_{U}<P<1$, the degree of variation is higher. The specific process is shown in Figure 3.

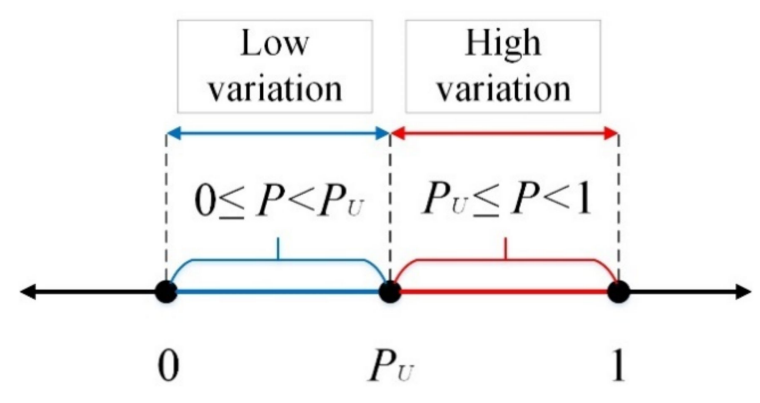

Figure 3. Variation range.

According to Figure 3, when $P_{U}<P<1$ at time $T_{i}$ and the degree of variation $P_{U}$ exceeds the variation threshold, a derived subtopic is generated. Here, the variation threshold $P_{U}$ is 0.8 .

\subsection{The Propagation Process of Derived Subtopics}

After the derived subtopics are generated, the popularity of the initial public opinion has not completely disappeared. Therefore, the information covered by the derived subtopics and the initial public opinion information are disseminated throughout the network at the same time. In turn, the multi-layer SIR model is used to simulate the propagation process. It is assumed that when an individual's attention to a subtopic is greater than a certain threshold, individuals participate in the discussion of the subtopic at that level. At the same time, the information propagation parameters of the new subtopics and the number of individuals participating in the discussion have changed, so this section defines these two parameters.

\subsubsection{The Propagation Parameter of Derived Subtopics}

When derived subtopics are generated, it means that a new topic begins to spread, and whether it can form derived public opinion is closely related to its propagation parameters and the number of individuals participating in the discussion. Consequently, Brownian motion is introduced to allow for random disturbance to the original public opinion 
parameters, which determines the information propagation parameters of the derived subtopics.

Definition 1 [32]. $(\Omega, F, P)$ is a probability space. If an adaptation process $B_{t}$ satisfies the following conditions in this probability space, $B_{t}$ is called Brownian motion or the Wiener process. For almost all $(\omega \in \Omega)$, the sample $B_{t}$ is continuous, and $B_{0}=0$.

(1) For all real numbers $s, t$ satisfies $0 \leq s \leq t, B_{t}-B_{s}$ and $F_{s}$ are independent;

(2) When $0 \leq s \leq t, B_{t}-B_{s}$ obeys the normal distribution $N(0, t-s)$, the normal distribution satisfies the mean value of 0 and the variance is $t-s$.

Since the subtopic is closely related to the initial public opinion, its propagation parameters are related to the initial public opinion propagation parameters. Here, the infection rate and recovery rate parameters of the $i$ th derived subtopic are defined by the following Formulas (4) and (5):

$$
\begin{aligned}
& \alpha_{i}=\alpha_{0}+\sigma_{i} \dot{B}_{i}(t), i=2,3, \ldots \\
& \beta_{i}=\beta_{0}+\sigma_{i} \dot{B}_{i}(t), i=2,3, \ldots
\end{aligned}
$$

where $B_{i}(t)$ is the independent Brownian motion, $\alpha_{0}$ and $\beta_{0}$ is the infection rate and immunity rate of the initial public opinion, respectively, and $\sigma_{i}>0\left(0<\sigma_{i}<1\right)$ is a constant, which represents the amount of information of the current subtopic, that is, the intensity of the disturbance. The greater the intensity, the greater the change in infection rate.

\subsubsection{The Propagation Model of Derived Subtopics}

After the derived subtopics are generated, different people will show different degrees of attention when facing the same hot topic. This difference in attention is a manifestation of the heterogeneous characteristics of individual [33] and it will affect whether the individual participates in the discussion of subtopics. Individuals' attention to derived subtopics is often affected by two factors. The first of these is the degree of relevance between the subtopic and the topic that formed the initial public opinion. Generally speaking, the higher the degree of relevance between the subtopic and its correlation, the more attention the individual pays to the subtopic. The second factor is the external influence from the individual's surrounding environment. If more people discuss the subtopic in the individual's surrounding environment, it is easier for the individual to pay attention to the subtopic.

The topic correlation and the number of infected neighbors $k_{i n f}$ are used to describe the degree of individual attention to derived subtopics. Setting $k_{\text {inf }}$ as the number of neighboring nodes infected around a node with degree $k$, then the attention degree function of a node with degree kin the network [34] is expressed by the following Formula (6):

$$
g_{i}=1-(1-\theta)^{k_{\text {inf }}}
$$

where the topic correlation $\theta \in(0,1)$. Generally speaking, when the correlation between the subtopic and the initial topic is 0 , it means that the subtopic is completely irrelevant to the initial topic. At this time, the subtopic cannot be regarded as a derived subtopic of the initial topic, so the individual attention is set to 0 at this time. When the relevance is 1 , it means that the subtopic is completely related to the initial topic. At this time, the relevant content of the subtopic is equivalent to the initial topic, so the individual attention is set to 1 . In addition, a greater degree of correlation between the subtopic and the initial topic means more individual attention given to the subtopic. The higher number of infections in the neighboring nodes around the individual means that the individual receives more information and consequently pays more attention to subtopics.

Since the number of infected neighbors around each individual is different and their number will change over time, the attention of the individual is also dynamically changing, 
that is, when a derived subtopic is generated at time $T_{i}$, the individuals with high attention to that subtopic will continue to participate in the propagation of the subtopic, while individuals with lower attention will lose interest in the subtopic and will no longer participate in the discussion of the subtopic, resulting in reduced topic spread. It is defined that when $g_{i}>g_{0}$ at the time $T_{i}$, individuals in the network will participate in the discussion of the subtopic and carry out the propagation of the subtopic. These individuals propagate according to the SIR model with the new propagation parameters and are intertwined with the initial public opinion to form a multi-layer SIR propagation network. The schematic diagram of their propagation transformation is shown in Figure 4.

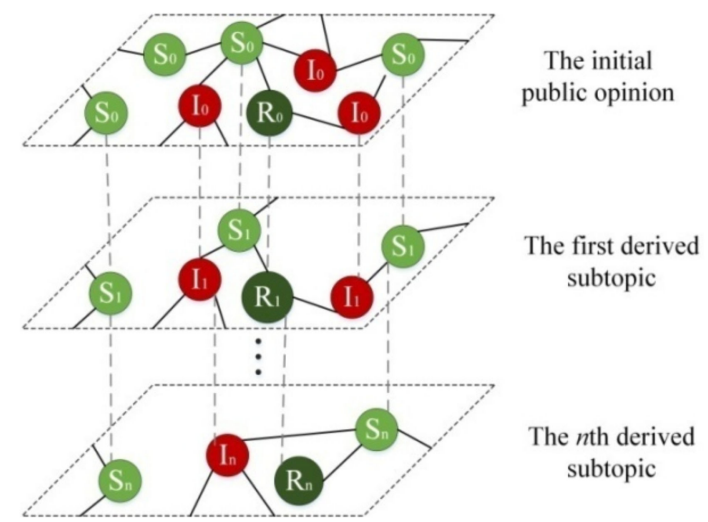

Figure 4. Propagation transformation of derived subtopics.

It can be seen from Figure 4 that the spread of initial public opinion corresponds to the first-level SIR communication network. After the first derived subtopic is generated, individuals in the network choose whether to participate in the discussion of the first derived subtopic according to their degree of attention. A second-layer SIR propagation network emerges, and the process goes on until $n$ subtopics are derived, when an $(n+1)$-layer SIR propagation network is formed, corresponding to the propagation process of $(n+1)$ topics.

\subsection{The Formation of Multi-Dimensional Derived Public Opinion}

Though derived subtopics have been generated and spread, not all subtopics will form derived public opinion. Therefore, this paper introduces the basic reproduction number from infectious disease modeling as the criterion for judging whether a given subtopic forms public opinion. The basic reproduction number refers to the number of people who can be infected by an infective person on average in an environment where all people are susceptible without intervention [35], and its expression is given in the following Formula (7) [36]:

$$
R_{0}=\left(1+\frac{r}{\alpha}\right)\left(1+\frac{r}{\beta}\right)
$$

Formula (7) is calculated by the new infection number data and transmission parameters at each time point, these being based on the above transmission dynamics model, where $r$ refers to the growth rate of the number of new infections at time $T_{i}$, and $\alpha$ and $\beta$ refer to the infection rate and immunity rate, respectively. In the infectious disease model, an important critical point of $R_{0}$ is $R_{0}=1$. The larger the value of $R_{0}$ is, the more difficult it is to control the epidemic. When $R_{0}<1$, the infectious disease will gradually disappear. When $R_{0}=1$, the infectious disease will become endemic. When $R_{0}>1$, infectious diseases will spread exponentially. According to the propagation of derived subtopics, the basic reproduction number $R_{0}$ of each derived subtopic is dynamically changing. It is defined here that when the basic reproduction number $R_{0}$ of the subtopic is larger than 1 and lasts for a period of time, the subtopic forms a network public opinion, i.e., a dimension is added to the initial public opinion. 
According to the above analysis, the specific simulation process of multi-dimensional public opinion generation is shown in Figure 5.

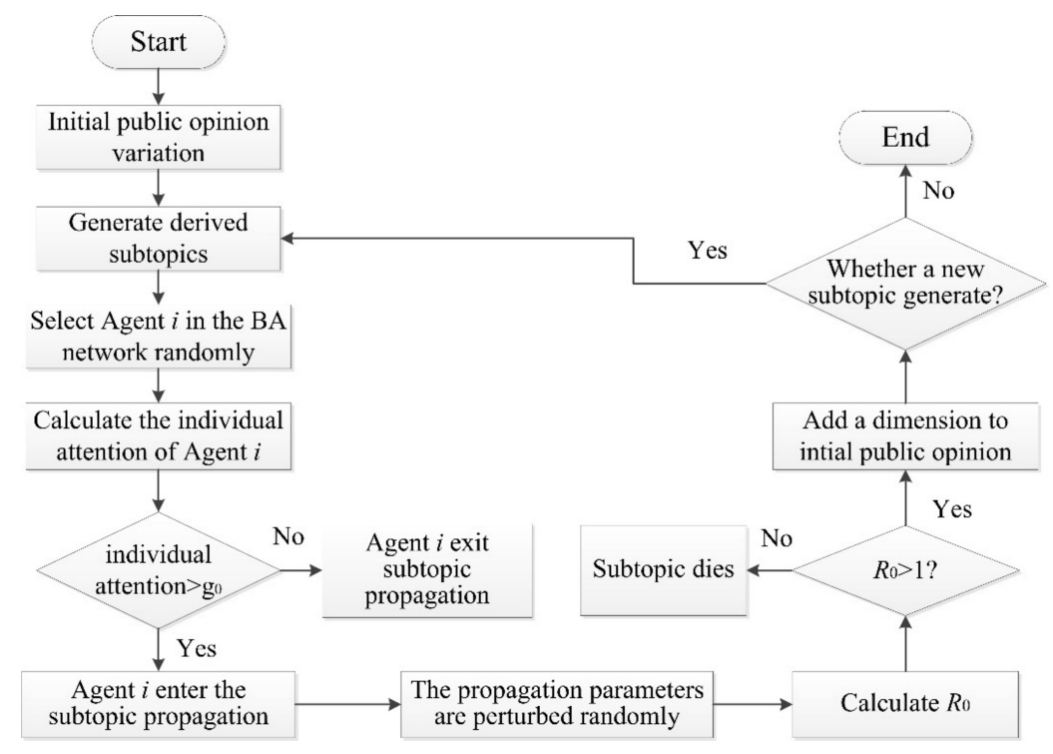

Figure 5. Multi-dimensional public opinion generation.

It can be seen from Figure 5 that when a derived subtopic is generated, the attention of an individual in the network to the subtopic is calculated at that moment. If it is greater than the attention threshold, the individual participates in the discussion of the subtopic. At the same time, the propagation parameters of the subtopic change, and the basic regeneration number also changes accordingly. When the basic reproduction number $R_{0}>1$, it is considered that the subtopic forms a derived public opinion. When multiple derived subtopics form a derived public opinion, this and the initial public opinion form a multi-dimensional public opinion.

\section{Simulation Experiments}

In this section, combined with the multi-dimensional public opinion formation model built above, we discuss the influence of the degree of information alienation, environmental force, topic correlation, and the amount of information contained in subtopics on the formation process of multi-dimensional public opinion. The degree of information alienation and environmental force are the initial influencing factors on derived subtopics, and the generation of derived subtopics is the basis for the formation of multi-dimensional public opinion. Therefore, when discussing these two factors, they are divided into two parts. The first is the influence on the number of derived subtopics and the propagation process. The second is the impact on the dimensions of multi-dimensional public opinion. In order to find the key factors that affect the dimensions of multi-dimensional public opinion, the above four factors are combined and compared, and finally the influence of different network topologies on the formation of multi-dimensional public opinion is further simulated.

\subsection{The Impact of Information Alienation on the Formation of Multi-Dimensional Public Opinion}

Based on the multi-dimensional public opinion formation model established in the previous section, initial parameters are set first. Assuming that in the initial state, only the initial public opinion is spread in the network, and the initial degree of variation is not 0 , at this time the derived subtopic has not yet been generated. Set the initial public opinion propagation parameters as: $\alpha=0.17, \beta=0.5$. The complex network chooses the BA (proposed by Barabasi and Albert) scale-free network [37]; the node size is set to $N=1000$. The relevant threshold is set to: $P_{U}=0.8, g_{0}=0.6$. In order to facilitate 
the observation of the formation of multi-dimensional public opinion, the subtopics are set to be derived from the variation of the initial public opinion information. At this time, each derived subtopic presents a positive correlation with the initial public opinion. Taking the comprehensive visualization into consideration, other parameters are set as: $\delta=9, \theta \sim N(0.9,0.3), \sigma \sim N(0.9,0.3)$, and the evolution time is set to $T=40$.

\subsubsection{The Impact of Information Alienation on the Amount of Derived Subtopics and} Propagation Process

When the information of the initial public opinion is varied, the degree of variation is higher and the degree of variation of the initial public opinion is greater. When the degree of variation reaches the threshold, derived subtopics are generated, and the longer the duration of the high variation state is, the more times the degree of variation reaches the threshold and the number of subtopics also increases. Based on this, this section will simulate and analyze the changes of variation under different information alienation settings. The results are shown in Figures 6 and 7.

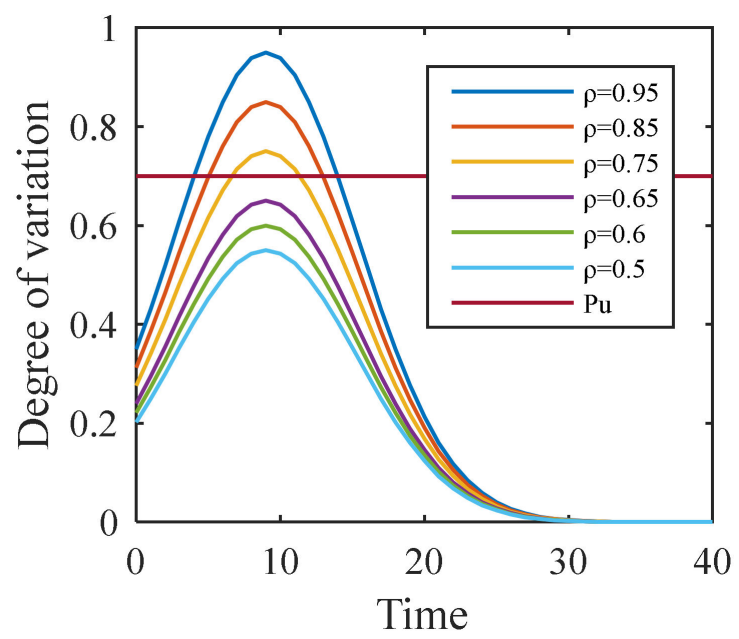

Figure 6. The changes of degree of variation under different information alienation settings.

It can be seen from Figure 6 that no matter how the degree of information alienation changes, the degree of initial public opinion variation always follows a similar trend, that is, as time goes by, the degree of variation will always reach a peak at the same time, and the overall trend will increase first and then decrease. In addition, when time $=0$, the initial public opinion variation degree is between 0.2 and 0.4 , indicating that the degree of information alienation has little effect on the degree of variation at the initial moment. As the degree of information alienation increases, the peak value of the degree of variation gradually increases. The degree of variation at the initial moment is almost unchanged. This shows that as the degree of information alienation increases, the degree of initial public opinion variation will reach the threshold of variation in a shorter time, resulting in the generation of new derived subtopics. Secondly, after the same evolution time, the stronger the degree of information alienation, the lower the initial public opinion is and the higher is the degree of variation. It can be seen from Figure 7 that when the degree of information alienation is lower than 0.75 , the number of derived subtopics generated is 0 , which means that only when the degree of information alienation reaches a certain value, there will be derived subtopics, and the number of derived subtopics increases with the deepening of the degree of information alienation. 


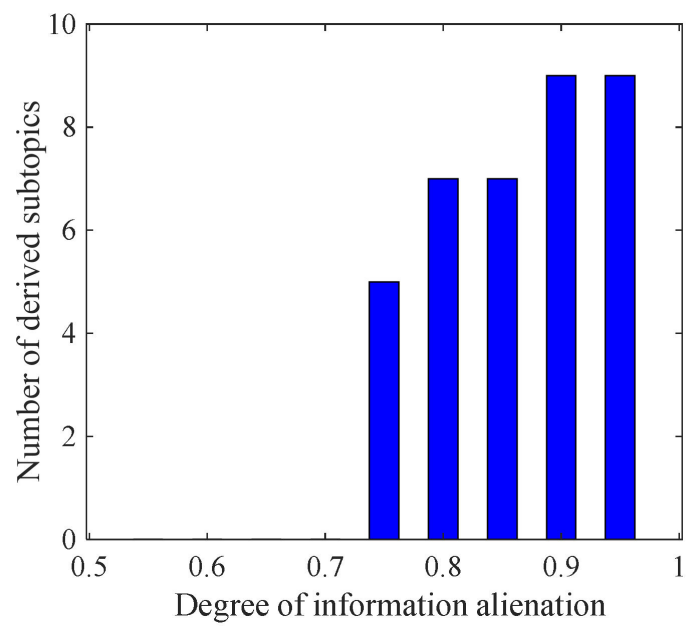

Figure 7. The number of derived subtopics under different information alienation settings.

In summary, the information alienation degree must reach a certain value before derived subtopics are generated. Therefore, the information alienation degree $\rho$ is set to 0.75 , 0.85 , and 0.95 respectively, and the derived subtopics generated are numbered according to the formation time, and the first is set as 1 . The influence of different information alienation settings on the propagation process of the subtopics is analyzed by analogy.

It can be seen from Figure 8 that with the increase in the degree of information alienation, the number of individuals discussing the derived subtopic 1 has not changed significantly, but the number of individuals discussing the derived subtopics 2,3 , and 4 has greatly increased. At $\rho=0.95$, the number of individuals discussing derived subtopics 2 and 3 is greater than that of derived subtopic 1 . The reason may be that the deviation caused by information alienation requires time to accumulate. Since the derived subtopic 1 is generated earlier and has a higher degree of overlap with the initial public opinion information, even if the degree of information alienation increases, the influence on the derived subtopic 1 will be small. At the same time, it shows that when the degree of information alienation is not high, most individuals tend to pay attention to the earliest derived subtopics and participate in the spread of subtopics. When the degree of information alienation is high, the number of derived subtopics generated is also large enough. The derived subtopic with the highest individual participation may not be the one that broke out first. In addition, it can be seen from Figure 8 that as the degree of information alienation increases, the number of derived subtopics further increases.

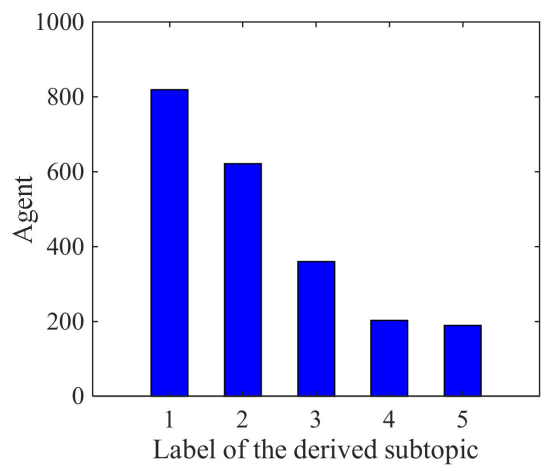

(a)

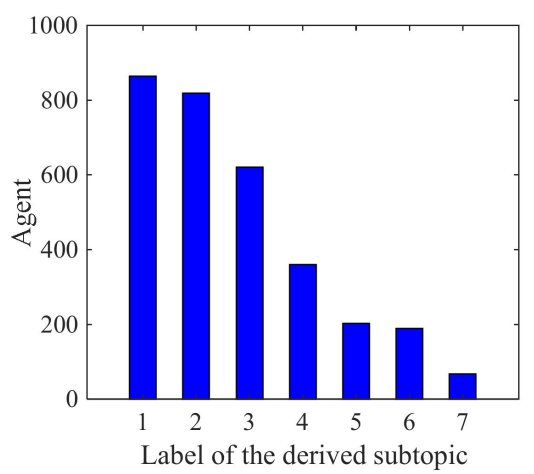

(b)

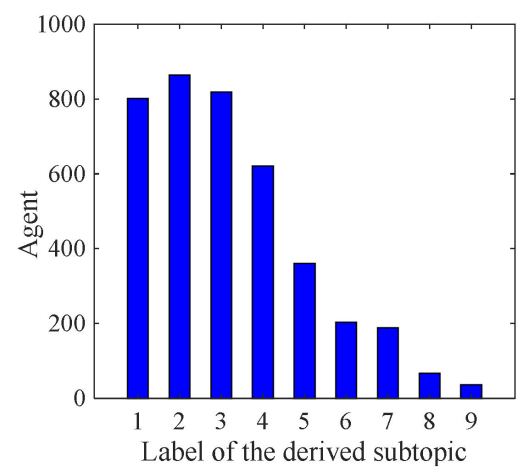

(c)

Figure 8. The number of agents participating in subtopic communication under different information alienation. (a) $\rho=0.75$. (b) $\rho=0.85$. (c) $\rho=0.95$. 
It can be seen from Figure 9 that the blue, red, and green lines represent the number of agents who are susceptible, infective, and recovered, respectively. Comparing the curve of the number of susceptible persons and the number of infective persons with respect to the initial public opinion and the subtopics in Figure 9, it can be seen that although the initial number of susceptible agents discussing subtopics is less than the number of those discussing the initial topics, the number of infective agents discussing some subtopics is the same as that discussing the initial topics. This indicates that the popularity is the same as the initial public opinion. With the deepening of information alienation, the number of such subtopics further increases.

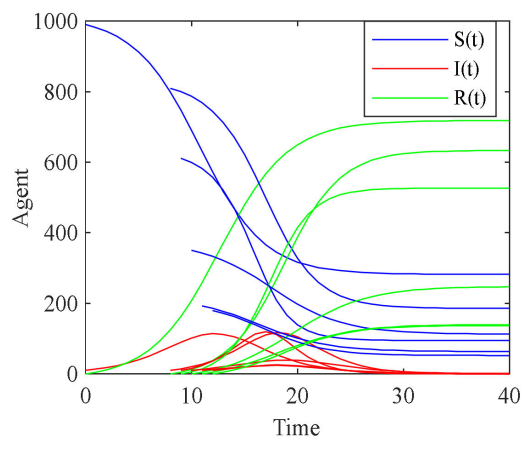

(a)

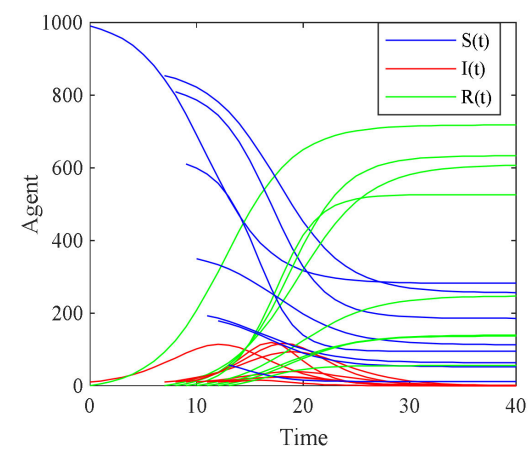

(b)

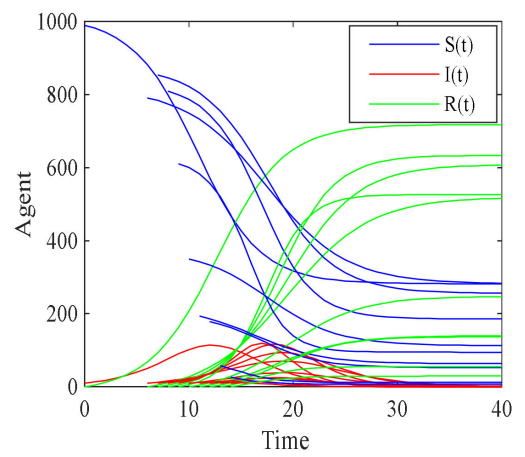

(c)

Figure 9. Evolution of initial public opinion and subtopics under different information alienation settings. (a) $\rho=0.75$. (b) $\rho=0.85$. (c) $\rho=0.95$.

4.1.2. The Impact of Information Alienation on the Degree of Multi-Dimensional Public Opinion

The different degree of information alienation affects the time for the generation of subtopics, the number of subtopics and the number of individuals participating in the discussion of the subtopics, which will affect the formation of subsequent multidimensional public opinions. Based on this, this section simulates the formation process of multi-dimensional public opinion under different degrees of information alienation and the results are shown in Figures 10 and 11.

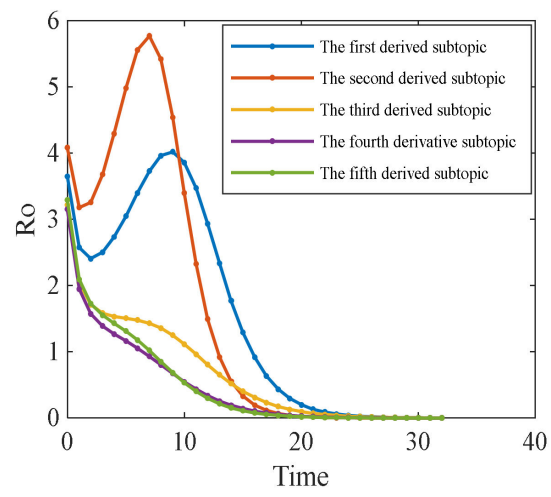

(a)

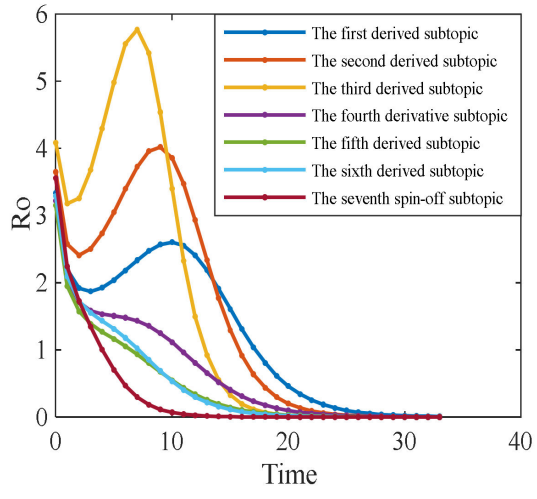

(b)

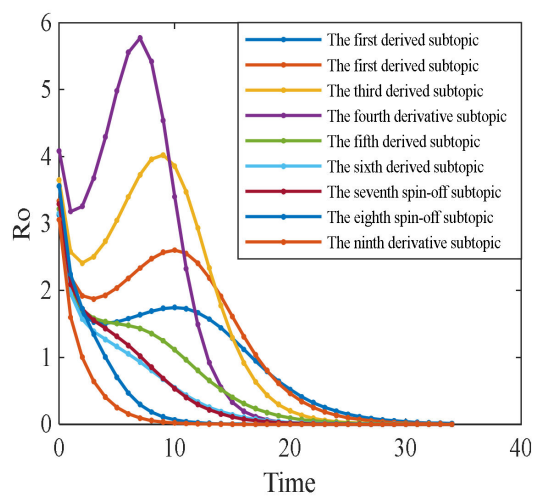

(c)

Figure 10. Changes of subtopics' $R_{0}$ values under different information alienation settings. (a) $\rho=0.75$. (b) $\rho=0.85$. (c) $\rho=0.95$. 


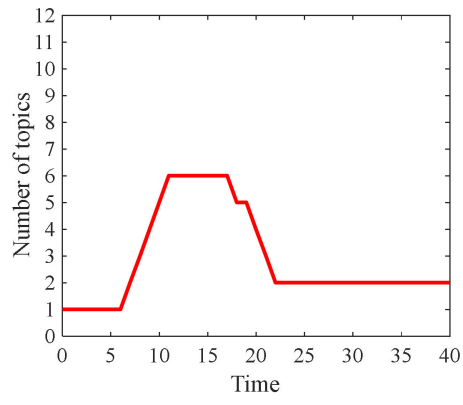

(a)

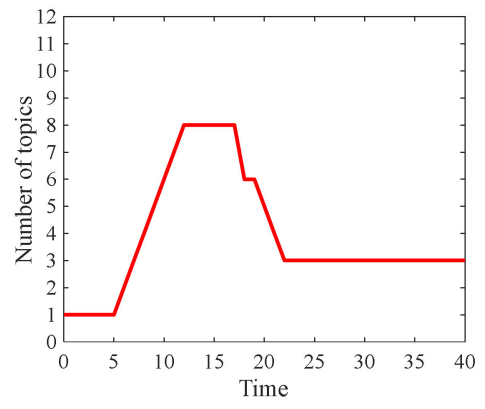

(b)

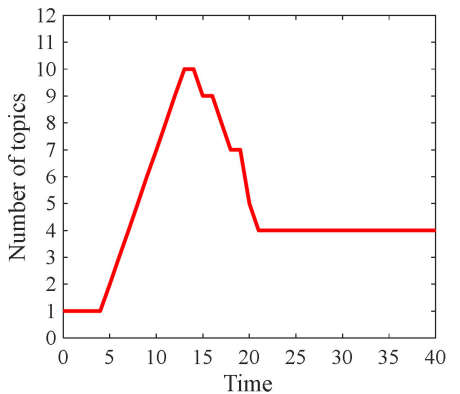

(c)

Figure 11. Changes of the number of topics under different information alienation settings. (a) $\rho=0.75$. (b) $\rho=0.85$. (c) $\rho=0.95$.

It can be seen from Figure 10 that the changes in the basic reproduction numbers of each derived subtopic under different information alienation settings shows two trends: one is a short-term decline in the initial stage, then a rise to a peak, and finally a decline and stabilization at 0 ; the other is a gradual or slow decrease and final stabilization at zero. This shows that after the emergence of subtopics, there has not yet been a largescale discussion in the initial stage, so the basic reproduction number declines. With the increase of netizens' participation in discussions, some subtopics may become hot topics due to the large amount of information or the influence of factors such as the network environment. Therefore, the basic reproduction number rapidly increases. At the same time, some subtopics may disappear as the initial basic reproduction number declines. In addition, with the increase of the degree of information alienation, the explosive growth of the basic reproduction number of the first derived subtopic becomes weaker and weaker. This shows that when the degree of information alienation is high, the earliest derived subtopics may not necessarily become explosive hot topics, but the derived subtopics generated in the middle and later stages are more likely to become hot topics.

In addition, it can be seen from Figure 11 that the number of topics are all 1 in the initial state. From time $=5$, derived subtopics are successively generated, and the number of topics also rapidly increases. With further propagation, some subtopics disappeared, and the number of topics also gradually decreases. In the end, some derived subtopics form derived public opinion, and the dimensions of multi-dimensional public opinion also stabilize at a certain value. Comparing Figure $10 a-c$, it can be seen that as the degree of information alienation increases, the number of topics starts to rise earlier, and as the number of subtopics increases, the peak of the number of topics rises from six to ten. The increase is large, but the final dimensions of the stable state has changed slightly, rising from two to four, which shows that the degree of information alienation has an impact on the number of derived subtopics, but it has a greater impact on the final public opinion dimension. The degree of influence is relatively small.

\subsection{The Impact of Environmental Forces on the Formation of Multi-Dimensional Public Opinion}

Environmental force $\delta$ refers to the influence of the spread of events or network communication channels on public opinion. Different social hot events receive different environmental forces, and the degree of information variation will be different. The number of derived subtopics generated, along with the formation process of multi-dimensional public opinion, will change accordingly, so the formation process of multi-dimensional public opinion under different environmental forces is analyzed. In order to describe the impact of different environmental forces on social hot events, set $\delta=3, \delta=5, \delta=7, \delta=9$, and the other parameters as: $\alpha=0.17, \beta=0.5, R=0.9, P_{U}=0.7, g_{0}=0.6, \rho=0.85, T=40$. 
4.2.1. The Impact of Environmental Forces on the Number of Derived Subtopics and the Propagation Process

Generally speaking, the greater the environmental impact of the initial public opinion, the higher the degree of variability, and the number of derived subtopics will increase accordingly. Based on this, the generation process of derived subtopics under different environmental forces is analyzed and the specific results are shown in Figures 12-15.

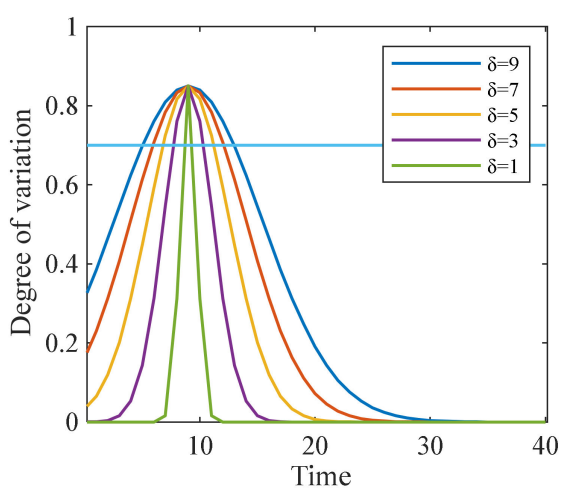

Figure 12. Changes of degree of variation under different information alienation settings.

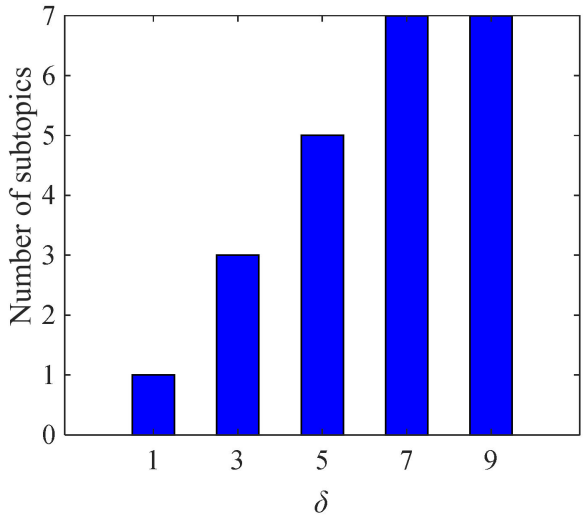

Figure 13. The number of derived subtopics under different information alienation settings.

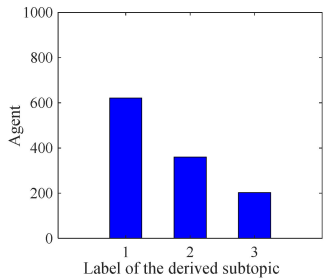

(a)

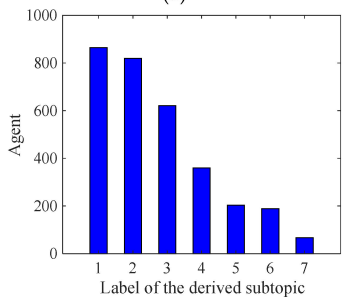

(c)

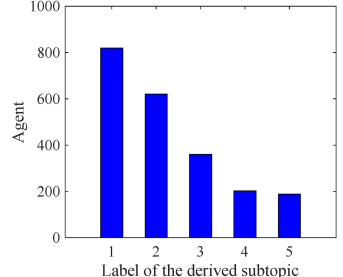

(b)

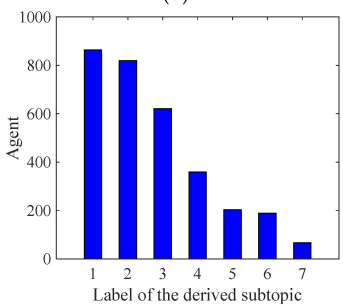

(d)

Figure 14. The number of agents participating in subtopic communication under different environmental forces. (a) $\delta=3$. (b) $\delta=5$. (c) $\delta=7$. (d) $\delta=9$. 


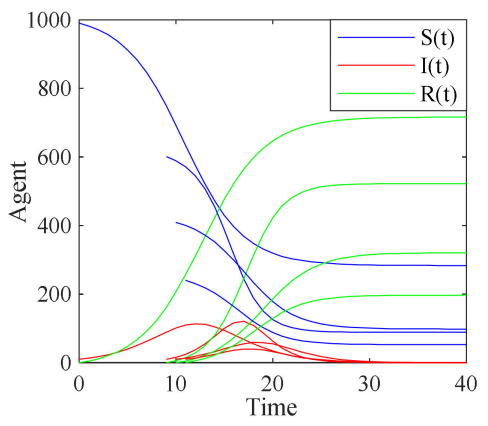

(a)

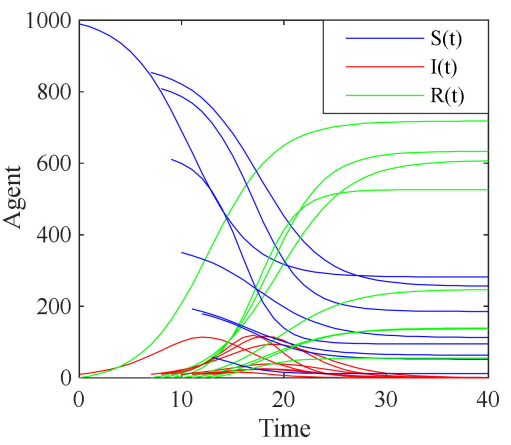

(c)

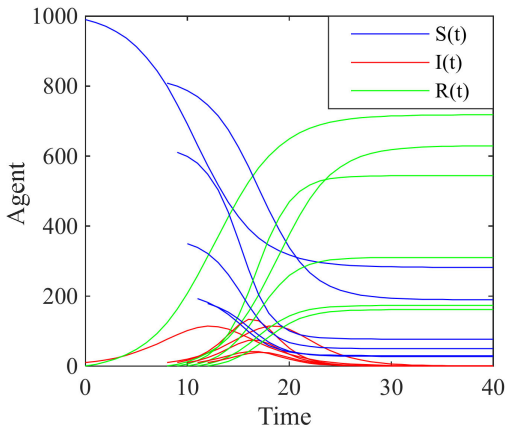

(b)

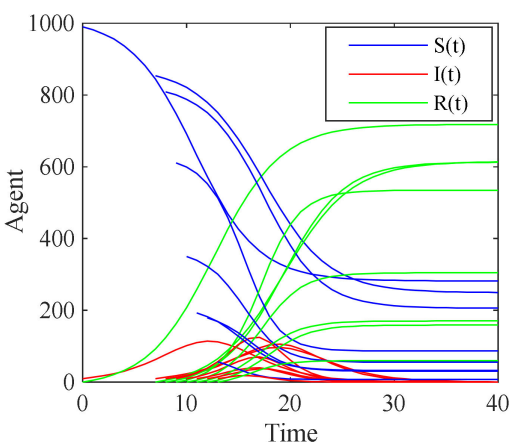

(d)

Figure 15. Evolution of initial public opinion and subtopics under different environmental forces. (a) $\delta=3$. (b) $\delta=5$. (c) $\delta=7$. (d) $\delta=9$.

It can be seen from Figure 12 that when the environmental force changes, the variation at the initial moment gradually increases but the peak value of the variation is basically the same; and when the environmental force is very small, the variation can still reach the threshold at a certain moment. Environmental forces have great impact on the initial degree of variation of social hot events, but little impact on the degree of variation in the evolution of initial public opinion. It can be seen from Figure 13 that as the environmental force increases, the number of derived subtopics increases. Even if the environmental force is only 1 , there are still derived subtopics. However, when the value reaches a certain level, the number of derived subtopics reaches a stable level. In addition, it can be seen from Figure 14 that with the increase of environmental forces, the number of individuals discussing derived subtopics does not change significantly, and, the earlier derived subtopics are generated, the more individuals participate in the discussion. With the increase of environmental forces, the number of individuals participating in each derived subtopic increases. In addition, it can be seen from Figure 15 that when the environmental force expands from 1 to 3 , the number of susceptible agents participating in the communication of the subtopic increases significantly.

However, when the environmental force continues to increase, the numbers of susceptible and infective people do not change significantly. This indicates that when the environmental force reaches a certain value, its influence on the spread of subtopics is small.

4.2.2. The Impact of Environmental Forces on the Dimensions of Multi-Dimensional Public Opinion

Different environmental forces change the time point, the number of subtopics, and the number of agents participating in the discussion of the subtopics, which further affect the subsequent formation of derived public opinion. Based on this, the basic reproduction number $R_{0}$ and the multidimensional public opinion dimensions of the subtopics under different environmental forces are simulated here and the results are shown in Figures 16 and 17. 


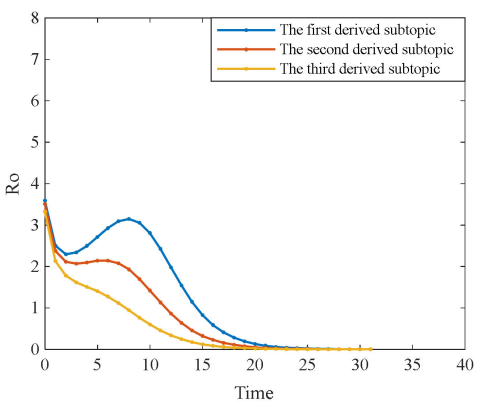

(a)

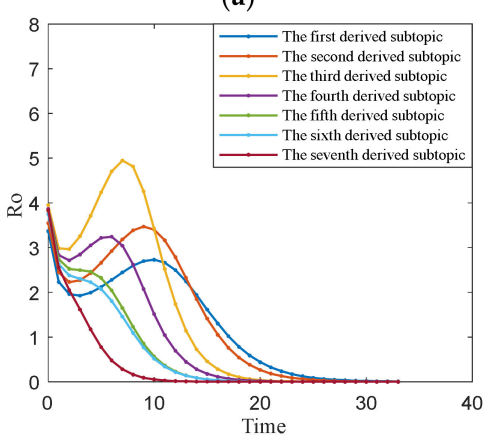

(c)

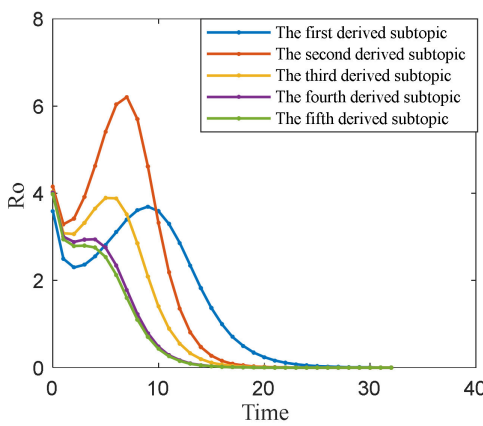

(b)

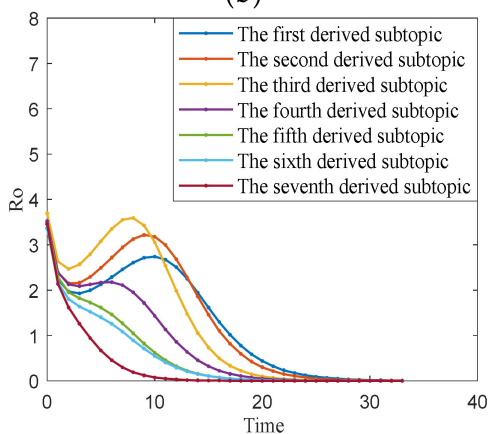

(d)

Figure 16. The change of $R_{0}$ under different environmental forces. (a) $\delta=3$. (b) $\delta=5$. (c) $\delta=7$. (d) $\delta=9$.

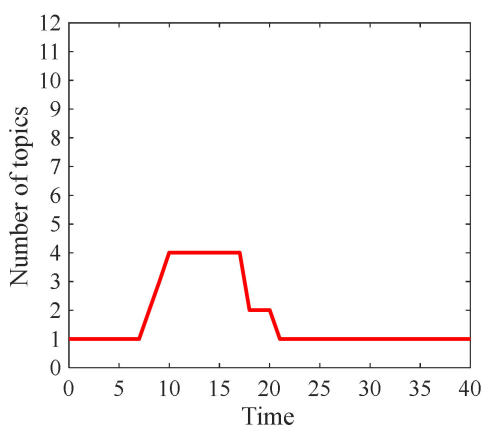

(a)

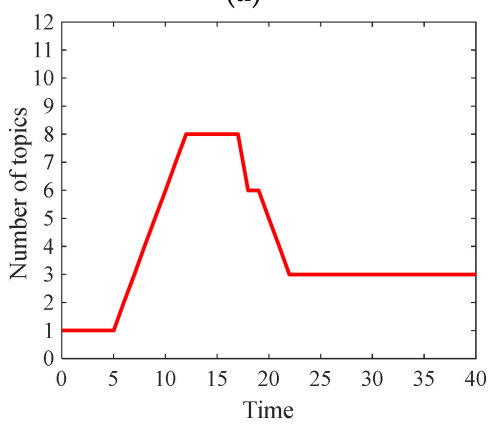

(c)

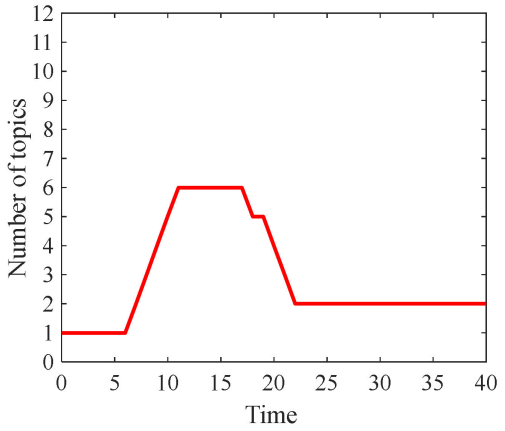

(b)

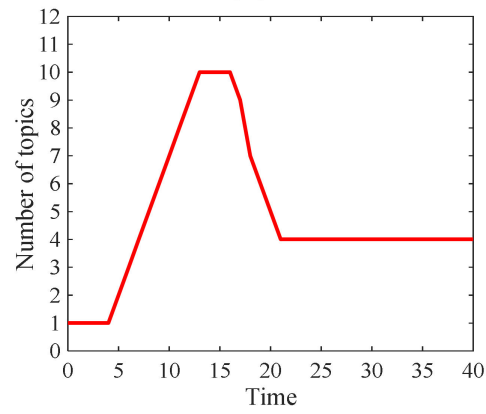

(d)

Figure 17. The change of the number of subtopics under different environmental forces. (a) $\delta=3$. (b) $\delta=5$. (c) $\delta=7$. (d) $\delta=9$.

It can be seen from Figure 16a-d that with the increase of environmental forces, the evolution trend of the basic regeneration number curve corresponding to derived subtopic 1 hardly changes, while the basic regeneration number of the second subtopic changes from the continuous decrease to growth, and the peak of $R_{0}$ reaches 6 . However, due to the 
increasing environmental force, the peak of the second derived topic is 3 . This shows that the environmental force has a relatively small impact on the first derived subtopic, and it has a greater impact on the second and third derived subtopics, and the basic regeneration number of the second subtopic increases with the increase in environmental force. However, when the environmental force increases to a certain level, the increase in the basic regeneration rate begins to slow down. From Figure 17, it can be seen that when the environmental force is 3 , although three derived subtopics are generated, the final public opinion dimension is still 1 , and no multi-dimensional public opinion is formed. At the same time, the increase of environmental forces has a positive correlation with the increase of the final public opinion dimension.

\subsubsection{Analysis of the Combination of Factors Influencing the Number of Derived Subtopics}

Through the above simulation analysis, it can be seen that the main factors affecting the number of derived subtopics are the degree of information alienation and the environmental forces. The derived subtopic as a transitional link in the formation of public opinion has a greater impact on its dimension, so these factors are combined for analysis. The result is shown in Figure 18.

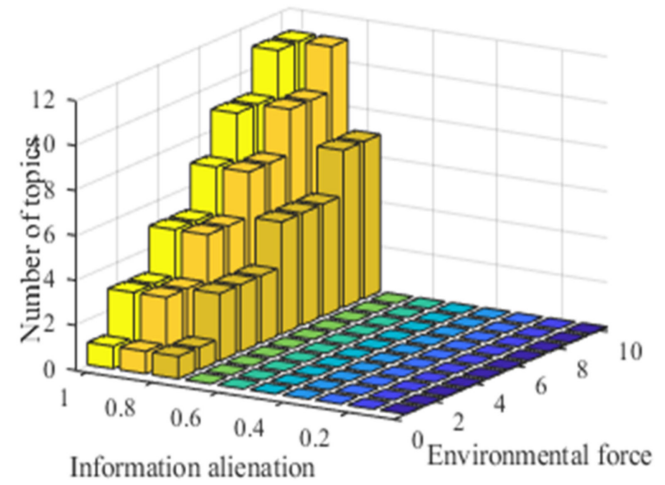

Figure 18. Analysis of the combination of factors influencing the number of derived subtopics.

It can be seen from Figure 18 that only when the degree of information alienation reaches a certain threshold will derived subtopics be generated. When the degrees of information alienation and environmental forces both increase, the number of derived subtopics increases to a certain extent, but the environmental force has a greater impact on the number of derived subtopics. When the degree of information alienation is 0.8 and the environmental force is 1 , the number of derived subtopics is 1 . When the degree of information alienation is unchanged and the environmental force is 9 , the number of derived subtopics is 7 , and the range of change is large. This shows that the degree of information alienation has an important impact on the formation of derived subtopics, and when there are derived subtopics, environmental forces will have more influence on the number of derived subtopics.

\subsection{The Impact of Topic Correlation on the Formation Process of Multi-Dimensional Public Opinion}

When the $i$ th derived subtopic is generated at time $T_{i}$, individuals in the network will pay attention to certain subtopics and participate in the discussion of that subtopic according to their own characteristics and environment. The degree of individual attention will be affected by the degree of correlation between the current subtopic and the initial public opinion and the number of surrounding infected neighbors. Therefore, by analyzing the degree of correlation between the subtopic and the initial public opinion, we can further explain which subtopics can form derived public opinions. The simulation results are as follows.

In order to explore whether subtopics that are highly related to the initial public opinion are more likely to form derived public opinions, a comparative analysis of Figures 19-21 
is carried out. When $\theta \sim N(0.3,0.3)$, it can be seen from Figure 19a that the first derived subtopic has the greatest correlation with the initial public opinion and the correlation with other subtopics is small. From Figures 20a and 21a, we can see that the peak value of the basic reproduction number $R_{0}$ of the first subtopic is also the highest, and the final public opinion dimension is 2 . When $\theta \sim N(0.5,0.3)$, the first and third derived subtopics have a relatively high relevance. From Figures $20 \mathrm{~b}$ and $21 \mathrm{~b}$, we can see that the peak value of the basic reproduction number $R_{0}$ of the first, second, and third subtopics is higher, and the final public opinion dimension is 4 . When $\theta \sim N(0.9,0.3)$, the correlation degree of each derived subtopic with the initial public opinion is relatively large, and the correlation degree of the second, fourth, sixth, and seventh subtopics even reaches 1 . From Figures 20c and 21c, the peak value of the basic reproduction number $R_{0}$ of the first, second, third, and fourth subtopics is higher, but the peak value reached by the curve has decreased, and the final public opinion dimension is 5 . When $\theta \sim U[0,1]$, the second, sixth, and seventh derived subtopics have a higher degree of correlation. From Figures $20 \mathrm{~d}$ and $21 \mathrm{~d}$, it can be seen that the peak value of the basic reproduction number $R_{0}$ of the second subtopic is higher. The final public opinion dimension is 4 . It can be seen from the above phenomenon that the peak of the basic reproduction number of topics with a high degree of correlation is correspondingly higher, which means that it is easier to form derived public opinion. When all the subtopics have a relatively high degree of correlation with the initial public opinion, the subtopic with the highest degree of correlation is relatively high. Topics may not be able to form derived public opinion, but the subtopics generated in the early stage are more likely to become hot topics and form derived public opinions. The reason may be that when the correlation of subtopics is relatively high, an individual will have a sense of freshness in the early subtopics, and as time goes by, they will gradually lose interest in the initial public opinion, even if there is a degree of correlation with initial public opinion in the later period. The subtopics with higher correlation are not very popular, leading to the failure to form a new public opinion.

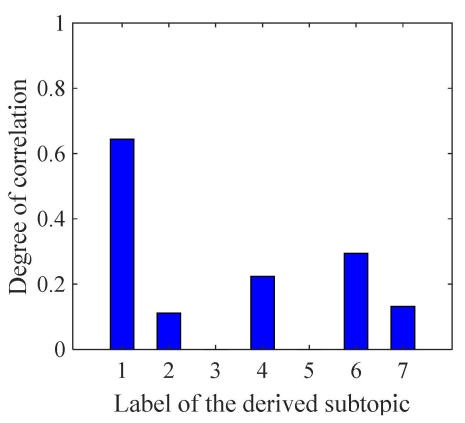

(a)

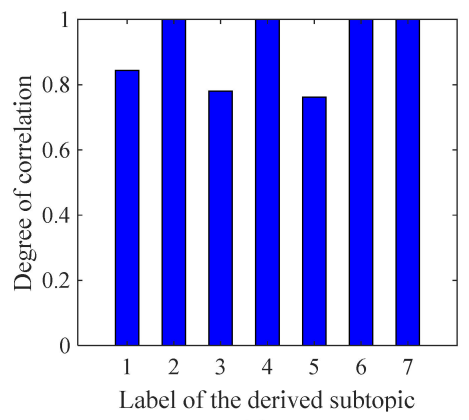

(c)

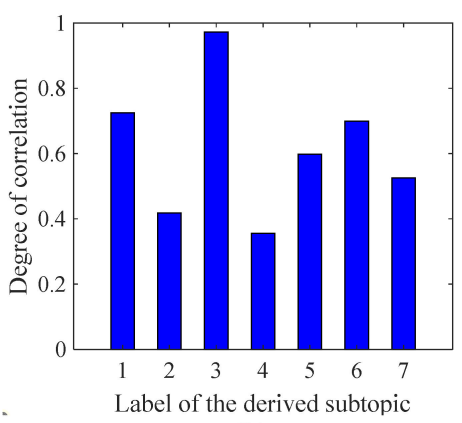

(b)

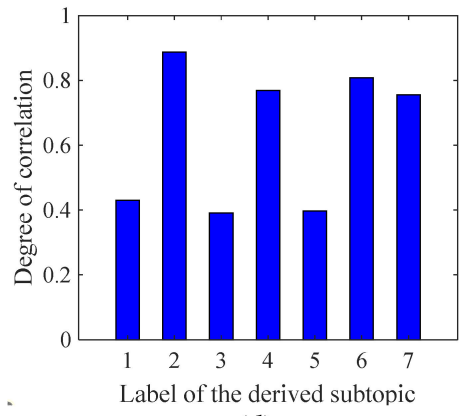

(d)

Figure 19. The correlation between derived subtopic number and initial public opinion. (a) $\theta \sim N(0.3,0.3)$. (b) $\theta \sim N(0.5,0.3)$. (c) $\theta \sim N(0.9,0.3)$. (d) $\theta \sim U[0,1]$. 


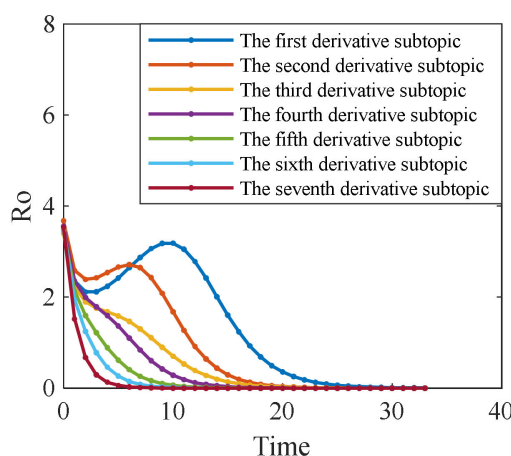

(a)

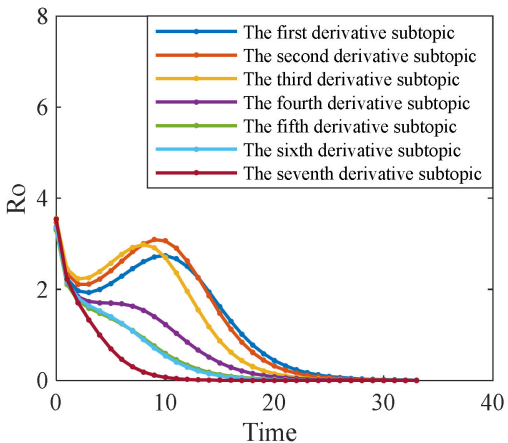

(c)

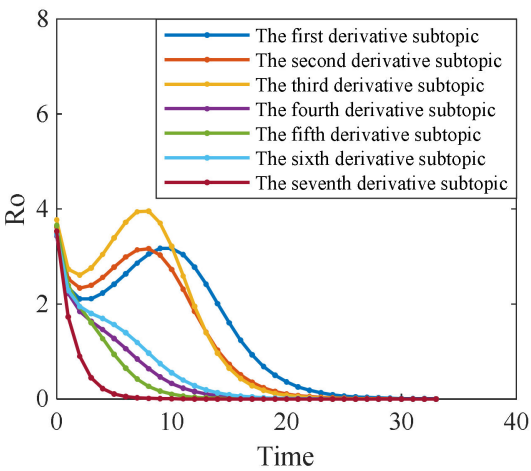

(b)

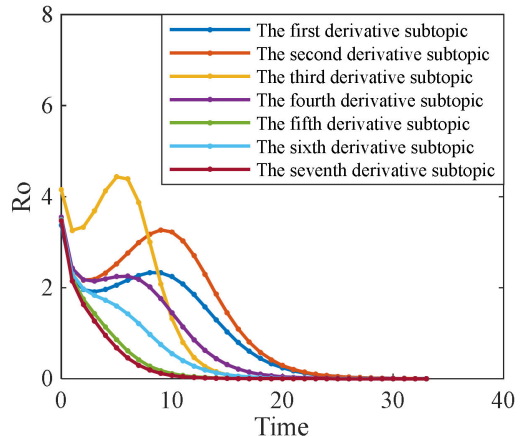

(d)

Figure 20. The change of $R_{0}$ under different distributions of correlation. (a) $\theta \sim N(0.3,0.3)$. (b) $\theta \sim N(0.5$ 0.3). (c) $\theta \sim N(0.9,0.3)$. (d) $\theta \sim U[0,1]$.

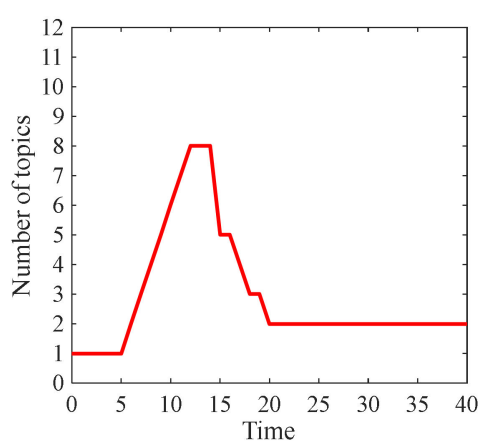

(a)

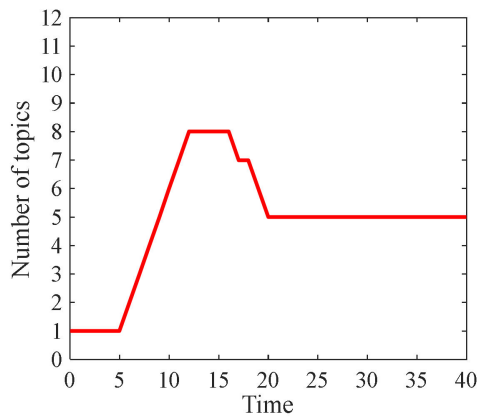

(c)

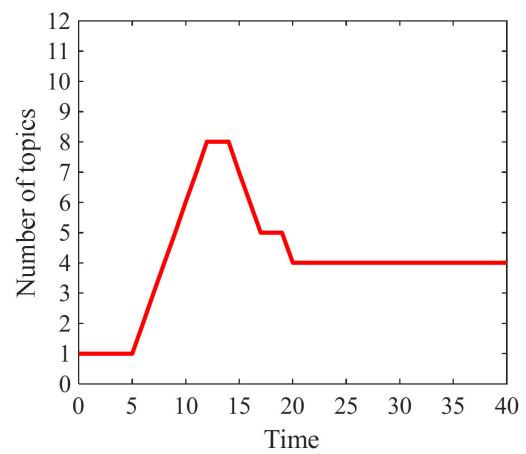

(b)

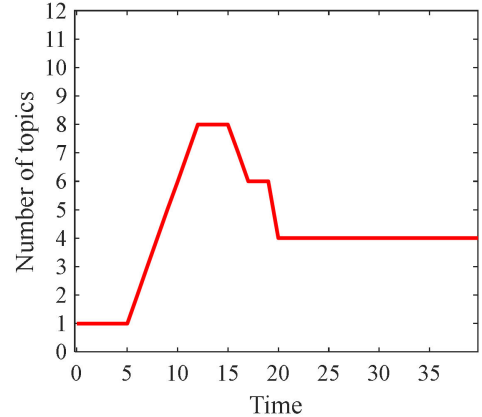

(d)

Figure 21. The change of the number of topics under different distributions of correlation. (a) $\theta \sim N(0.3,0.3)$. (b) $\theta \sim N(0.5,0.3)$. (c) $\theta \sim N(0.9,0.3)$. (d) $\theta \sim U[0,1]$. 
4.4. The Impact of the Amount of Information Contained in Subtopics on the Formation Process of Multi-Dimensional Public Opinion

After different derived subtopics are generated, the content posted by individuals participating in the topic discussion is different, and the amount of information contained is also different. In order to explore whether derived subtopics with a large amount of information are more likely to form derived public opinion, simulation analysis is used. The impact of the amount of information contained in subtopics on the formation of multi-dimensional public opinion is analyzed, and the specific results are shown in Figures 22-24.

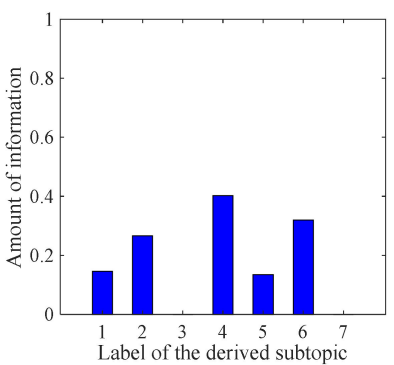

(a)

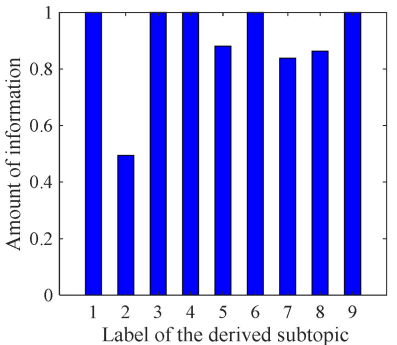

(c)

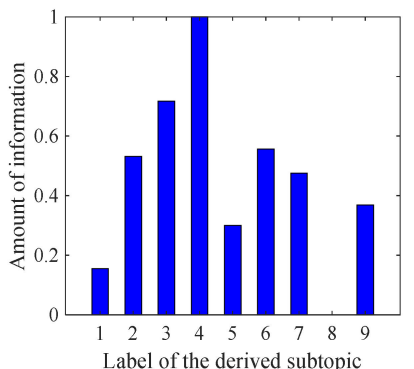

(b)

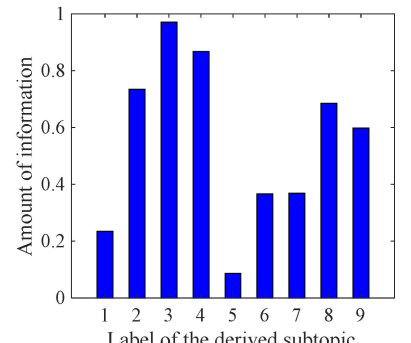

(d)

Figure 22. The amount of information of each subtopic under different distributions. (a) $\sigma_{i} \sim N\left(0.1\right.$. 0.3). (b) $\sigma_{i} \sim N(0.5,0.3)$. (c) $\sigma_{i} \sim N(0.9,0.3)$. (d) $\sigma_{i} \sim U[0,1]$.

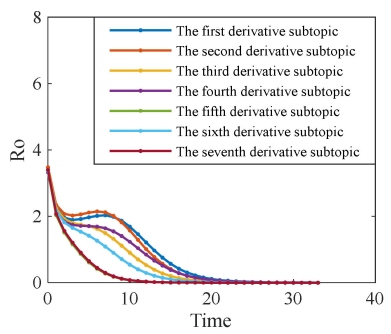

(a)

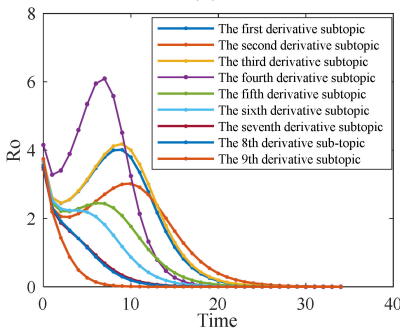

(c)

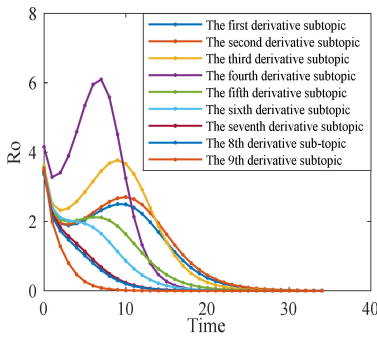

(b)

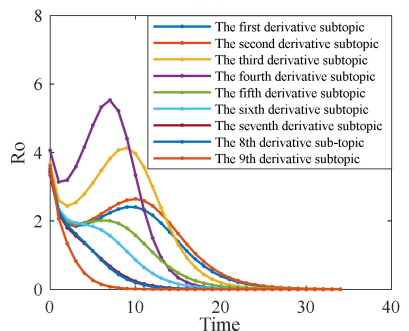

(d)

Figure 23. The change of $R_{0}$ under different distributions of the amount of information. (a) $\sigma_{i} \sim N(0.1,0.3)$. (b) $\sigma_{i} \sim N(0.5,0.3)$. (c) $\sigma_{i} \sim N(0.9,0.3)$. (d) $\sigma_{i} \sim U[0,1]$. 


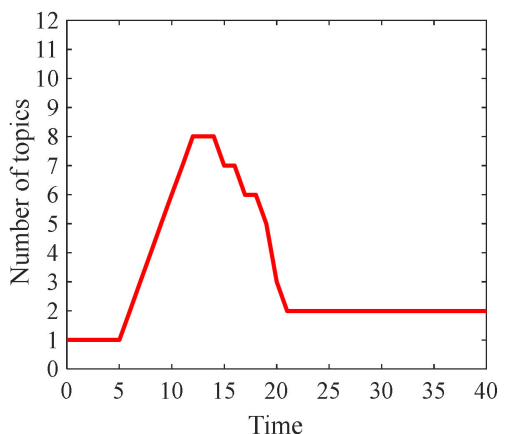

(a)

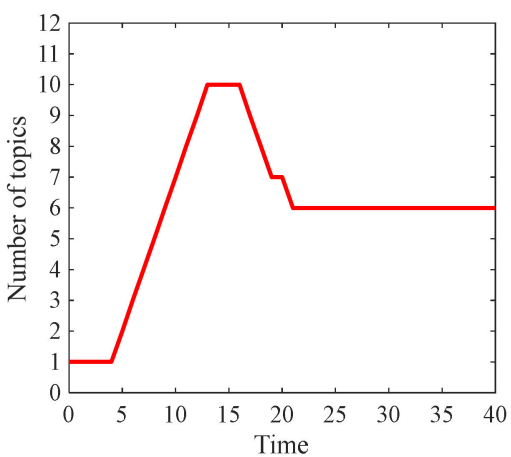

(c)

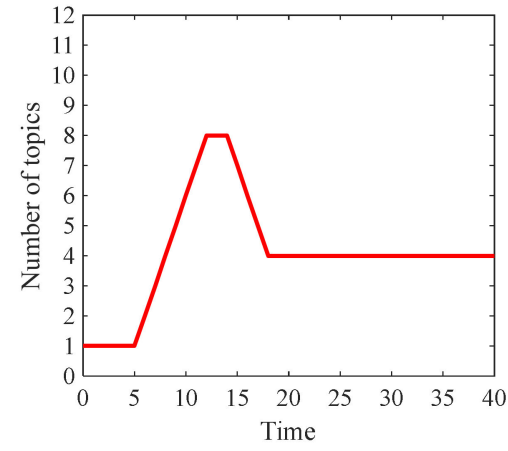

(b)

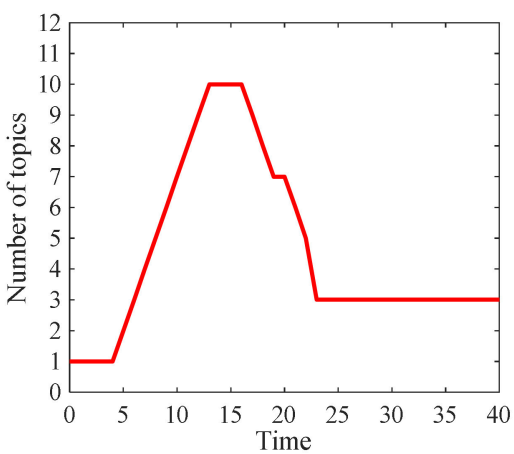

(d)

Figure 24. The change of the number of topics under different distributions of the amount of information. (a) $\sigma_{i} \sim N(0.1,0.3)$. (b) $\sigma_{i} \sim N(0.5,0.3)$. (c) $\sigma_{i} \sim N(0.9,0.3)$. (d) $\sigma_{i} \sim U[0,1]$.

In order to analyze whether subtopics with a large amount of information are more likely to form derived public opinion, a comparative analysis is carried out. When $\sigma \sim N(0.3,0.3)$, it can be seen from Figure 22a that the amount of information contained in all derived subtopics is relatively small. From Figures $23 \mathrm{a}$ and $24 \mathrm{a}$, we can see that the peak of $R_{0}$ is low, and the final public opinion dimension is only 2 . When $\sigma \sim N(0.5,0.3)$, the second and third derived subtopics contain more information. From Figures $23 \mathrm{~b}$ and 24b, it can be seen that the peak value of the basic reproduction number $R_{0}$ of the second and third subtopics is also the highest, and the final public opinion dimension is 4 . When $\sigma \sim N(0.9,0.3)$, each derived subtopic contains a large amount of information. Among them, the amount of information contained in the first, third, fourth, and ninth subtopics even reaches 1. From Figures $23 c$ and $24 c$, we can see that the peak value of the basic reproduction number $R_{0}$ of the first, third, fourth, and fourth subtopics is also higher, and the final public opinion dimension is 6 . When $\sigma \sim U[0,1]$, the third and fourth subtopics contain more information. From Figures $23 \mathrm{~d}$ and $24 \mathrm{~d}$, it can be seen that the peak of the basic reproduction number $R_{0}$ of the third and fourth subtopics is also higher, and the final public opinion dimension is 3 . From the above phenomenon, it can be seen that the peak value of the basic reproduction number $R_{0}$ of the subtopic with a large amount of information is correspondingly higher, and it is easier to form a new one-dimensional derived topic.

\subsection{Combination Analysis of Various Factors Affecting the Multi-Dimensional Public Opinion}

Through the above simulation analysis, it can be known that there are many factors that affect the dimensions of multi-dimensional public opinion. In the actual evolution of public opinion, due to the urgency of online public opinion control, it is usually necessary to focus on key links in order to reduce the cost of public opinion prevention and control. Therefore, finding the most critical factor from the many factors that affect the formation of multi-dimensional public opinion is more in line with realistic requirements. Therefore, we 
will find out the key factors through a combination analysis of the factors that affect the formation of multi-dimensional public opinion. The results are shown in Figures 25-28.

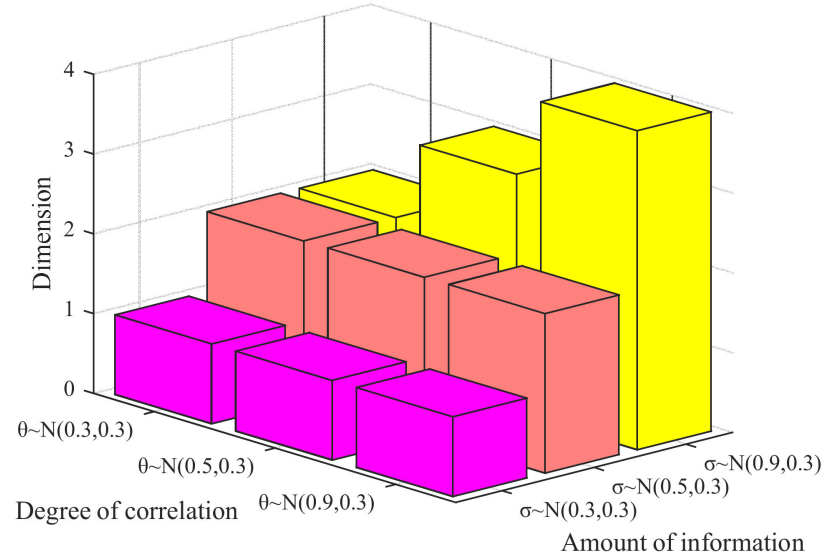

Figure 25. Relationship among degree of correlation, amount of information and dimension.

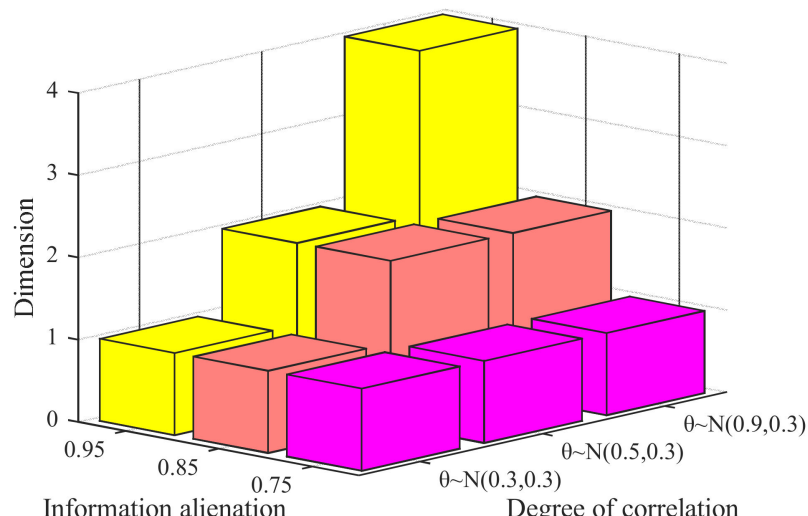

Figure 26. Relationship among degree of correlation, information alienation and dimension.

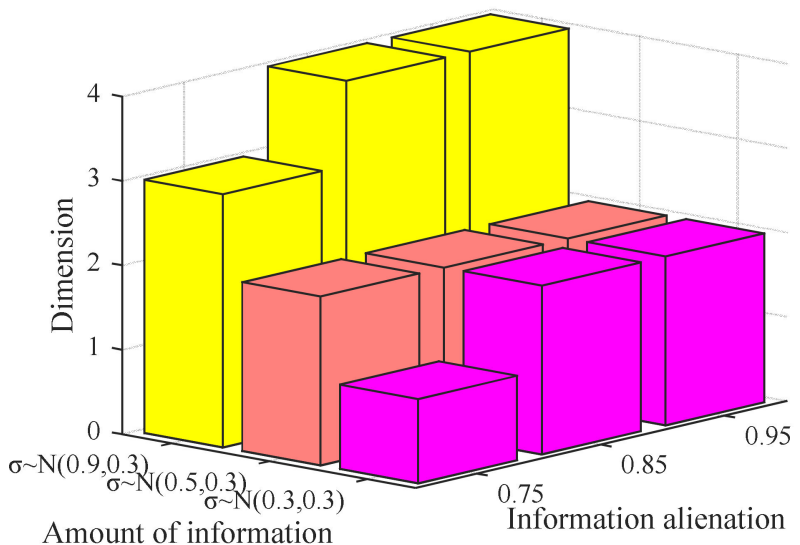

Figure 27. Relationship among amount of information, information alienation and dimension.

Figures 25-27 respectively show the relationship among degree of correlation, amount of information, and dimension, the relationship among degree of correlation, information alienation and dimension, the relationship among amount of information, information alienation and dimension. It can be seen from Figure 25 that when the amount of information contained in a subtopic is small, its correlation does not impact on the dimension. 
When the amount of information contained is large, as the correlation increases, the dimension increases. No matter how the degree of correlation changes, the dimensions of public opinion increase when the amount of information increases. Therefore, comparing the effect of correlation, the amount of information contained in subtopics has a greater impact on the dimension. It can be seen from Figure 26 that with the increase in the degree of correlation and information alienation, the dimension increases, and the increase in the two is not much different. It can be seen from this that: the degree of correlation and the degree of information alienation have little effect on the dimensions of public opinion. It can be seen from Figure 27 that as the degree of information alienation and the amount of information contained in subtopics increase, the dimensions of public opinion increase to a certain extent. When the amount of information is $\sigma \sim N(0.5,0.3)$, as the degree of information alienation increases, the dimensions of public opinion do not change. When the degree of information alienation increases with the amount of information contained in the subtopics, the dimensions of public opinion change. It can be seen that, compared to the degree of information alienation, the amount of information contained in subtopics has a greater impact on the dimensions of public opinion.

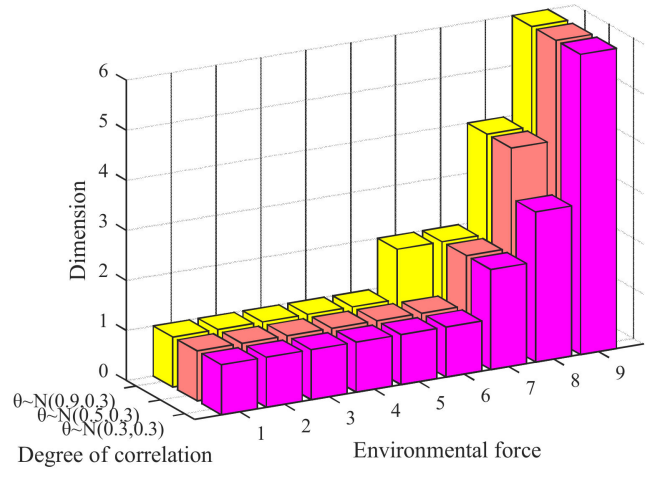

(a)

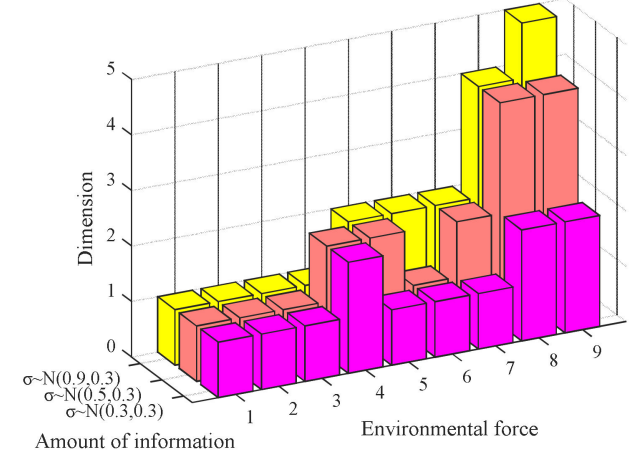

(b)

Figure 28. Combination analysis of environmental forces, correlation and the amount of information contained in subtopics. (a) The relationship among dimension, environmental forces and correlation. (b) The relationship among dimension, environmental forces and amount of information.

In addition, Figure 28 shows the relationship among environmental forces, correlation and the amount of information contained in subtopics. From Figure 28a, it can be seen that when the environmental force is small, it has little effect on the dimensions of public opinion. When the environmental force is large, the dimensions of public opinion increase sharply with the increase of environmental force. With the increase of the degree of correlation, the dimensions of public opinion have not changed or only increased slightly. Therefore, compared with the degree of correlation, the influence of environmental forces on the dimensions of public opinion is greater. From Figure 28b, it can be seen that as the amount of information contained in the subtopics and the environmental forces increase, the dimensions of public opinion increase significantly. Therefore, the amount of information and environmental forces contained in the subtopics impact more on the dimensions of public opinion. Based on the above combination analysis, the amount of information contained in subtopics and environmental forces are the key factors that affect the dimensions of public opinion.

\subsection{The Influence of Network Topology on the Formation Process of Public Opinion Dimensions}

Differences in network structure have an important influence on the formation and evolution of public opinion. Therefore, it is necessary to simulate and analyze the formation process of multi-dimensional public opinion under different network topologies. Since the previous simulation analysis is based on the BA network, the influence of different network topologies on the dimensions of public opinion is studied. The BA network, a fully 
connected network and a WS small world network are selected for comparative analysis. The network topology parameters are as follows.

It can be seen from Table 3 that the aggregation coefficient and average of the fully connected network are larger than other networks, but the average path length is smaller. Other parameters are set as follows: $\rho=0.85, \delta=8, \sigma \sim N(0.5,0.3), \theta \sim N(0.9,0.3), P_{U}=0.7$, $g_{0}=0.6, N=1000$. The simulation results are shown in Figures 29 and 30 .

Table 3. Different network topology parameters.

\begin{tabular}{cccc}
\hline Network Name & Average Path Length & Aggregation Coefficient & Average \\
\hline WS small world network & 5.3719 & 0.0088 & 4 \\
\hline BA network & 4.0282 & 0.034 & 3.964 \\
\hline Fully connected network & 1 & 1 & 999 \\
\hline
\end{tabular}

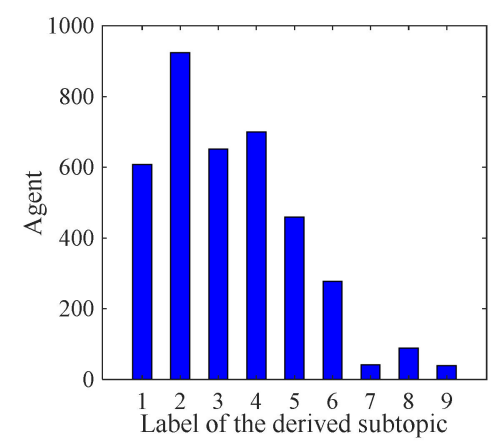

(a)

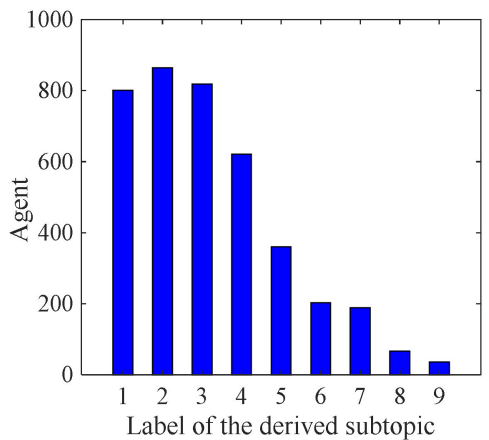

(b)

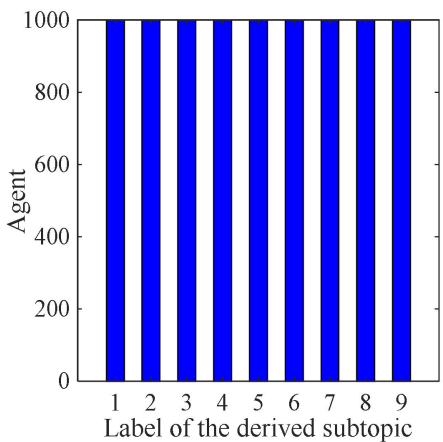

(c)

Figure 29. The number of agents participating in the discussion of derived subtopics under different network topologies. (a) WS small world network. (b) BA network. (c) Fully connected network.

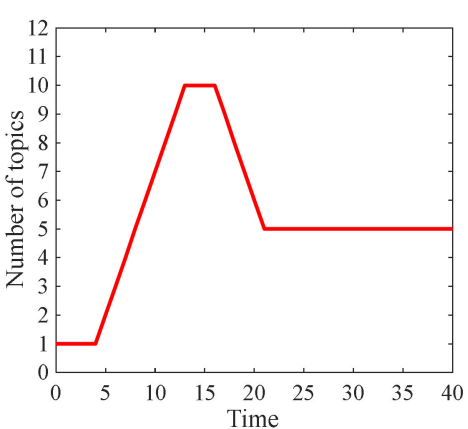

(a)

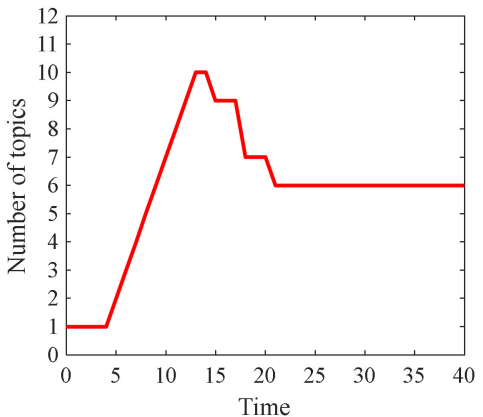

(b)

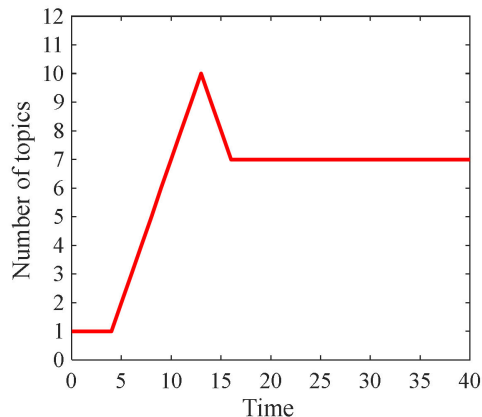

(c)

Figure 30. The change of number of topics under different network topologies. (a) WS small world network. (b) BA network. (c) Fully connected network.

It can be seen from Figure 29 that 9 derived subtopics are generated under different network structures. In addition, in the fully connected network, the number of agents participating in the discussion of each derived subtopic is 1000, indicating that the participation of each subtopic is very high. While in the WS small world network and the BA network, only part of the subtopics' participation is high, which means that the network topology does not have much impact on the number of subtopics, it has a greater impact on the number of agents participating in the discussion of the subtopics. In a fully connected network, the participation of each derived subtopic is up to the highest. As can be seen from Figure 30, in different network structures, the dimensions ultimately formed by multidimensional public opinion are different. Fully connected networks will eventually form 
the highest dimensions, and WS small world networks will eventually form the lowest dimensions. This change is related to network topology parameters. The change of the aggregation coefficient is the same, which is opposite to the change of the average path length. Therefore, it can be seen that the multi-dimensional public opinion formed by the network topology with a high aggregation coefficient and short average path length has a higher dimension.

\section{Empirical Analysis}

The news of COVID-19 that broke out in late 2019 swept across China and the world. At the same time, hot events related to the epidemic are fermenting on the Internet, and the spread of some rumors has caused many netizens to panic. After a long period of antiepidemic work, the epidemic situation in China has basically stabilized and people's lives have gradually returned to normal. However, on 9 November 2020, China's CCTV news broadcast "One new local confirmed case of COVID-19 in Shanghai" ignited netizens' panic. With the development of the Shanghai epidemic, the incident has spawned many topics, and even the discussion on the derived topics has surpassed that of the original topics. The original topic of "One new local confirmed case of COVID-19 in Shanghai" derived further subtopics, such as "Shanghai Epidemic Prevention and Control Work Conference", "One Community in Pudong New Area will be Upgraded to Medium Risk Degree", and "4015 people in Shanghai Pudong Hospital have been quarantined". Discussions on these derived subtopics have even pushed the public's panic to a climax. Here, we searched the Baidu index with "Shanghai epidemic" as the key word and obtained the search index from 18 November to 9 December 2020, as shown in Figure 31.

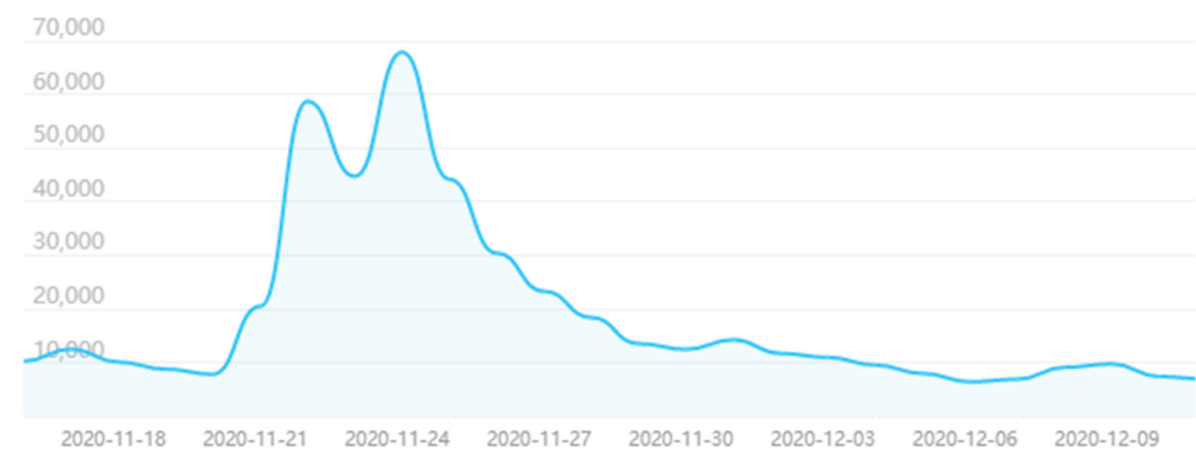

Figure 31. "Shanghai epidemic" Baidu index.

It can be seen from Figure 31 that the "One new local confirmed case of COVID-19 in Shanghai" reached the highest search interest degree from November 21 to 24, and then stabilized, and most derived subtopics were generated during this time period, indicating that network public opinion has an important influence on the formation and propagation of derived subtopics.

In order to study the phenomenon of the formation of multi-dimensional public opinion for the above-mentioned events, event-related microblogs released by media outlets such as "Tou tiao", "The Paper News", and "CCTV News" between 9 November and 29 November 2020 are collected. The results are shown in Table 4.

It can be seen from Table 4 that after the outbreak of the original topic "One new local confirmed case of COVID-19 in Shanghai", with the development of the incident, 11 derived subtopics were successively formed and the amount of reading and discussion of each derivative subtopic was different. The distribution map of the number of individuals participating in the discussion is simulated based on the time when the derived subtopics are generated and the number of discussions among netizens. It also defines that if the total number of readings of a derived subtopic is greater than 100 million and the total number of discussions by netizens is greater than 5000, the subtopic is regarded as a derived public 
opinion, that is, a dimension is added to the initial public opinion, and the time cut-off point is set by December 9th. Based on this simulation, the public opinion dimension change map of the "One new local confirmed case of COVID-19 in Shanghai" event was generated and the result is shown in Figure 32.

Table 4. Topic related to "Shanghai epidemic" public opinion.

\begin{tabular}{|c|c|c|c|c|}
\hline Release Time & Topic & Reading Volume & Discussion Volume & Topic Number \\
\hline 11.09 & $\begin{array}{l}\text { \#One new local confirmed case of COVID-19 } \\
\text { in Shanghai \# }\end{array}$ & $240 \mathrm{~m}$ & 6607 & 0 \\
\hline 11.10 & $\begin{array}{l}\text { \#Shanghai Epidemic Prevention and Control } \\
\text { Work Conference\# }\end{array}$ & $300 \mathrm{~m}$ & 18,000 & 1 \\
\hline 11.21 & $\begin{array}{l}\text { \#One Community in Pudong New Area Was } \\
\text { Upgraded to Medium Risk Degree\# }\end{array}$ & $23.868 \mathrm{~m}$ & 594 & 2 \\
\hline 11.21 & $\begin{array}{c}\text { \#One Community in Pudong New Area Will } \\
\text { Be Upgraded to Medium Risk Degree } \\
\text { Tomorrow\# }\end{array}$ & $130 \mathrm{~m}$ & 3452 & 3 \\
\hline 11.21 & $\begin{array}{l}\text { \#4015 people in Shanghai Pudong Hospital } \\
\text { have been quarantined\# }\end{array}$ & $450 \mathrm{~m}$ & 17,000 & 4 \\
\hline 11.21 & $\begin{array}{l}\text { \#83 people that once contacted with infected } \\
\text { person were tracked\# }\end{array}$ & $100 \mathrm{~m}$ & 6383 & 5 \\
\hline 11.21 & $\begin{array}{l}\# 1 \text { new COVID-19 case confirmed } \\
\text { among15,416 people in Shanghai\# }\end{array}$ & $32.914 \mathrm{~m}$ & 1319 & 6 \\
\hline 11.23 & $\begin{array}{l}\text { \#2 new local confirmed cases of COVID-19 in } \\
\text { Shanghai\# }\end{array}$ & $710 \mathrm{~m}$ & 28,000 & 7 \\
\hline 11.23 & $\begin{array}{l}\text { \#One COVID-19 patient once exposed toan } \\
\text { aviation container\# }\end{array}$ & $310 \mathrm{~m}$ & 8109 & 8 \\
\hline 11.29 & \#Shanghai Songjiang \# & $12.469 \mathrm{~m}$ & 3106 & 9 \\
\hline 11.29 & \#No COVID-19 in Shanghai Songjiang \# & $6.118 \mathrm{~m}$ & 431 & 10 \\
\hline 1129 & $\begin{array}{c}\text { \#Reasults of } 6 \text { local confirmed case of } \\
\text { COVID-19 in Shanghai\# }\end{array}$ & $190 \mathrm{~m}$ & 3670 & 11 \\
\hline
\end{tabular}

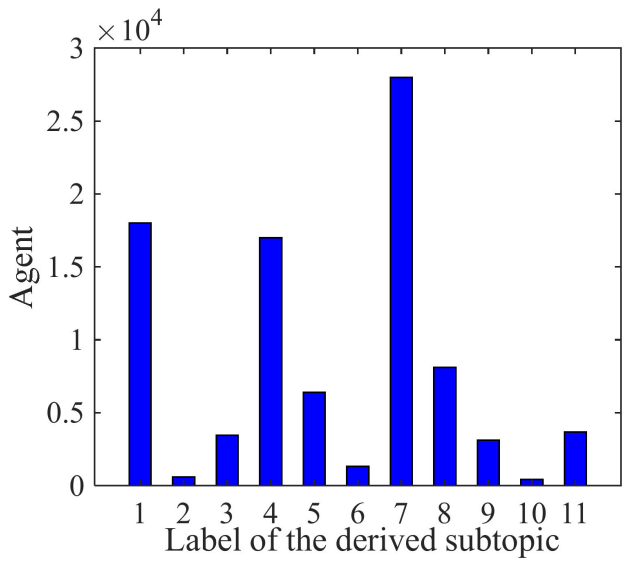

(a)

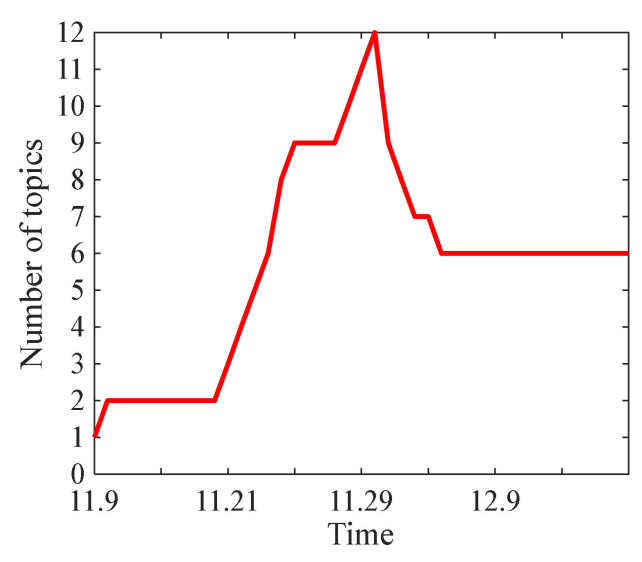

(b)

Figure 32. Simulation of derived subtopics for this public opinion. (a)The numberof agents discussing derived subtopics. (b) Change inthe number of topics.

It can be seen from Figure 32a that the number of agents associated with the first, fourth, and seventh derived subtopics is relatively large, which means that participation is high. It can be seen from Figure 32b that the dimensions of public opinion began to 
rise on November 21, reached a peak on the 29th, and finally stabilized at six dimensions, and formed a six-dimensional public opinion. This six-dimensional public opinion is the original public opinion.

The following is a simulation of the event based on the multi-dimensional public opinion formation model mentioned in this paper. Due to the large amount of case data and comprehensive visualization considerations, the simulation network scale is set to 1000. Since there are many derived subtopics generated, settings were at $\rho=0.95, \delta=9$, and the amount of information contained in the subtopics $\sigma \sim N(0.9,0.3)$, the initial public opinion infection rate set at $\alpha=0.2, \beta=0.5$. The result is shown in Figure 33 .

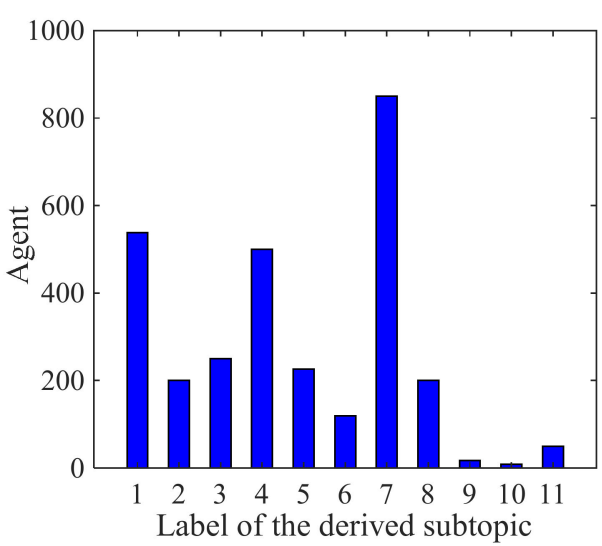

(a)

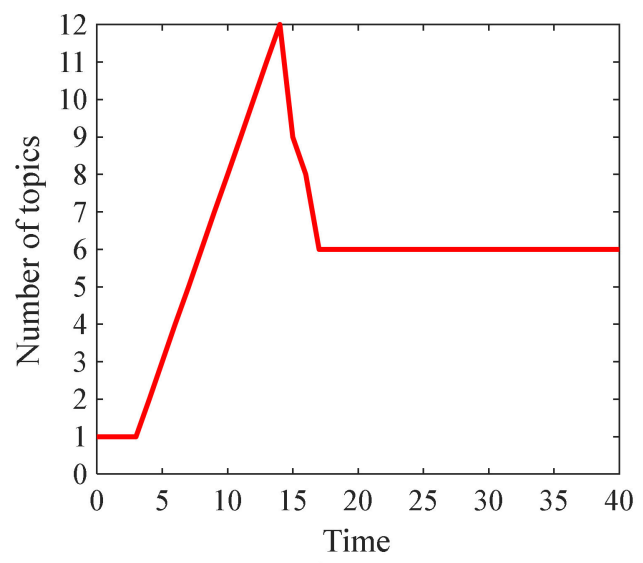

(b)

Figure 33. Simulation of derived subtopics for this public opinion used the model proposed in this paper. (a) The number of agents discussing derived subtopics. (b) Change in the number of topics.

It can be seen from Figure 33 that the simulation of the multi-dimensional public opinion using the model proposed in this paper is not much different from the actual results. First of all, it can be seen from Figure 33a that the number of individuals participating in the first, fourth, and seventh derived subtopics is the largest, which is consistent with reality. It can be seen from Figure 33b that the dimensions of public opinion reached up to twelve, then began to decline, and finally stabilized at six dimensions. Although the rise and decline process is slightly different from the reality, the overall trend is basically the same. In general, the multi-dimensional public opinion formation model proposed in this paper can better simulate social hot events in reality and has important guiding significance for analyzing the causes of multi-dimensional public opinion and predicting the evolutionary trends of multi-dimensional public opinion.

\section{Conclusions}

This paper discusses the formation process of multi-dimensional public opinion based on derived topics. Usually, after a hot news story breaks out, the social network platforms generate the initial topic of the event and form the initial public opinion as the discussion heats up. As time goes by, netizens' subjective understanding of the initial public opinion information will gradually become biased and gradually accumulate, leading to variation in the initial public opinion so that multiple subtopics are derived. After the subtopics are generated, the SIR model is used to analyze the propagation process and calculate the basic reproduction number to determine whether the derived public opinion can be formed, and this derived new public opinion is intertwined with the initial public opinion to form a multi-dimensional public opinion together. Finally, simulation experiments are conducted to explore the influence of factors, such as the degree of information alienation, environmental forces, topic correlation, and the amount of information contained in subtopics on the formation of multi-dimensional public opinion.

The following conclusions are obtained through simulation experiments: 
(1) When the degree of information alienation reaches a certain threshold, derived subtopics will be generated. In addition, when the degree of information alienation is high, the earliest derived subtopics may not necessarily form derived public opinion but later derived subtopics may also be generated. Derived public opinion is formed, and the degree of information alienation has a greater impact on the number of derived subtopics but has small impact on the dimensions of the final state of public opinion.

(2) Environmental forces and the amount of information contained in subtopics are the key factors that affect the formation of multi-dimensional public opinion. Among them, environmental forces have a greater impact on the early subtopics, and the amount of information contained in subtopics is key to forming derived public opinion.

(3) Subtopics that are highly related to the initial public opinion topic are more likely to form derived public opinions. When all subtopics are highly correlated with the initial public opinion, the subtopic with the highest degree of correlation may not necessarily form a derived public opinion, but subtopics generated in the early stage are more likely to form a derived public opinion.

(4) The network topology does not have much impact on the number of subtopics, but it has a greater impact on the number of individuals participating in the discussion of the subtopics, and the dimensions of multidimensional public opinion formed by the network topology with a high aggregation coefficient and short average path length are greater.

However, this paper still has the following shortcomings, which will require further study:

(1) This paper does not consider the influence of external information intervention in the study of the formation process of multi-dimensional public opinion and subsequent intervention mechanisms which can be introduced to study the influence of external information on initial public opinion and derived public opinion.

(2) The paper considers the situation of static nodes without considering the increase or withdrawal of Internet users' nodes [38]. In reality, individuals participating in discussion of derived subtopics often increase and decrease. Therefore, the multidimensional public opinion evolution mechanism under the dynamic network can be considered in the follow-up research.

(3) In this paper, the dynamic equations for complex networks only considers the node average degree of uniform networks and cannot reflect the connections between each node and its neighbor. Therefore, more complex dynamic equations should be considered to simulate the infection process of each node in future.

Author Contributions: T.C. described the proposed framework and wrote the whole manuscript; X.Y. implemented the simulation experiments; J.Y. and G.L. collected the data; G.C. revised the manuscript. All authors have read and agreed to the published version of the manuscript.

Funding: This research is supported by the National Social Science Foundation of China (Grant No. 20BTQ059), the Project of China (Hangzhou) Cross-border E-commerce College (No. 2021KXYJ07), the Contemporary Business and Trade Research Center and Center for Collaborative Innovation Studies of Modern Business of Zhejiang Gongshang University of China (Grant No. 14SMXY05YB), as well as the Characteristic \& Preponderant Discipline of Key Construction Universities in Zhejiang Province (Zhejiang Gongshang University-Statistics).

Institutional Review Board Statement: Not applicable.

Informed Consent Statement: Not applicable.

Data Availability Statement: The data used to support the findings of this study are available from the corresponding author upon request.

Conflicts of Interest: The authors declare that they have no conflicting interest. 


\section{References}

1. An, L.; Dai, Y.; Zhou, Y. Research on the formation and evolution of public opinion derived from public safety events-based on topic and time series analysis. Public Secur. Stud. 2020, 12, 18-35.

2. Chen, T.; Rong, J.; Yang, J.; Cong, G.; Li, G. Combining Public Opinion Dissemination with Polarization Process Considering Individual Heterogeneity. Healthcare 2021, 9, 176. [CrossRef]

3. Lan, Y.; Dong, X.; Zeng, R.; Qi, Z. Research on effects model of derived of network public opinion from the perspective of information alienation. J. Intell. 2015, 34, 139-143.

4. Zhang, Y.; Feng, Y. Two-layer coupled network model for topic derivation in public opinion propagation. China Commun. 2020, 17, 176-187. [CrossRef]

5. Korobeinikov, A. Global properties of SIR and SEIR epidemic models with multiple parallel infectious stages. Bull. Math. Biol. 2009, 71, 75. [CrossRef] [PubMed]

6. Arenas, A.; Cota, W.; Gomez-Gardenes, J.; Gomez, S.; Steinegger, B. A mathematical model for the spatiotemporal epidemic spreading of COVID19. MedRxiv 2020, 10, 1101.

7. Estrada, E. COVID-19 and SARS-CoV-2. Modeling the present, looking at the future. Phys. Rep. 2020, 869, 1-51. [CrossRef]

8. Yang, L.; Li, Z.; Giua, A. Containment of rumor spread in complex social networks. Inf. Sci. 2020, 506, 113-130. [CrossRef]

9. Zanette, D.H. Dynamics of rumor propagation on small-world networks. Phys. Rev. E 2002, 65, 041908. [CrossRef]

10. Moreno, Y.; Nekovee, M.; Pacheco, A.F. Dynamics of rumor spreading in complex networks. Phys. Rev. E 2004, 69, 066130. [CrossRef]

11. Zhou, J.; Liu, Z.; Li, B. Influence of network structure on rumor propagation. Phys. Lett. A 2007, 368, 458-463. [CrossRef]

12. Tan, Y.; Lin, Q.; Luan, Y.; Chen, T.; Qiao, Y.; Luan, Y. Campus Network Public Opinion Monitoring System Based on Reptile Technology. IOP Conf. Ser. Earth Environ. Sci. 2019, 252, 21. [CrossRef]

13. You, C.; Zhu, D.; Sun, Y.; Ye, A. Social-Network-Oriented Public Opinion Monitoring Platform Based on ElasticSearch. Comput. Mater. Contin. 2019, 61, 1271-1283. [CrossRef]

14. Chen, T.; Yin, X.; Peng, L.; Rong, J.; Yang, J.; Cong, G. Monitoring and Recognizing Enterprise Public Opinion from High-Risk Users Based on User Portrait and Random Forest Algorithm. Axioms 2021, 10, 106. [CrossRef]

15. Wang, C.; Wang, G.; Luo, X.; Li, H. Modeling rumor propagation and mitigation across multiple social networks. Phys. A Stat. Mech. Its Appl. 2019, 535, 122240. [CrossRef]

16. Askarizadeh, M.; TorkLadani, B.; Manshaei, M.H. An evolutionary game model for analysis of rumor propagation and control in social networks. Phys. A Stat. Mech. Its Appl. 2019, 523, 21-39. [CrossRef]

17. Zhang, S. Multi-Dimensional Analysis of causes of network-derived public opinion from the perspective of spreading factors. J. Beijing Adm. Inst. 2019, 4, 56-63.

18. Duncan, J.; Peter, S. Influentials, networks, and public opinion formation. J. Consum. Res. 2007, 34, 441-458.

19. Li, H. Research on evolution law and countermeasure of the derived network public opinion based on information alienation theory-taking internet rumors governance for example. J. Mod. Inf. 2015, 35, 4-8.

20. Snyder, J.; Cai, W.; D'Souza, R. Degree-targeted cascades in modular, degree-heterogeneous networks. Phys. Soc. 2020, 1, 09316.

21. Wang, L.; Dai, J. Research on the quantitative analytical method of network public opinion derived chain. Inf. Sci. 2016, 34, 59-63.

22. Chen, T.; Li, Q.; Fu, P.; Yang, J.; Xu, C.; Cong, G.; Li, G. Public opinion polarization by individual revenue from the social preference theory. Int. J. Environ. Res. Public Health 2020, 17, 946. [CrossRef]

23. Li, J.; Xiao, R. Agent-based modelling approach for multidimensional opinion polarization in collective behaviour. J. Artif. Soc. Soc. Simul. 2017, 20, 14. [CrossRef]

24. Parsegov, S.E.; Proskurnikov, A.V.; Tempo, R.; Friedkin, N.E. Novel multidimensional models of opinion dynamics in social networks. IEEE Trans. Autom. Control 2016, 62, 2270-2285. [CrossRef]

25. Sun, Y.; Chai, R. An Early-Warning Model for Online Learners Based on User Portrait. Ingénierie Des Systèmesd' Inf. 2020, 25, 535-541. [CrossRef]

26. Wang, G.; Chi, Y.; Liu, Y.; Wang, Y. Studies on a multidimensional public opinion network model and its topic detection algorithm. Inf. Process. Manag. 2019, 56, 584-608. [CrossRef]

27. Wang, Y.; Wang, J. SIR rumor spreading model considering the effect of difference in nodes' identification capabilities. Int. J. Mod. Phys. C 2017, 28, 15. [CrossRef]

28. Lu, Y. Study on the Spread of Complex Network Virus Based on Human Behavior. Ph.D. Thesis, Nanjing University of Posts and Telecommunications, Nanjing, China, 2015.

29. Liu, D.; Wang, W.; Li, H. Evolutionary mechanism and information supervision of public opinions in internet emergency. Procedia Comput. Sci. 2013, 17, 973-980. [CrossRef]

30. Wang, H.; Lan, Y.; Pan, Y. Research on the causes of internet derived public opinion from the perspective of information alienation dynamics. Mod. Intell. 2013, 33, 59-63. [CrossRef]

31. Guo, Y. Analysis of Variation and Evolution of Network Public Opinion Based on Cellular Automata. Ph.D. Thesis, Beijing Jiaotong University, Beijing, China, 2016.

32. Liu, C. Random Process; Huazhong University of Science and Technology Press: Wuhan, China, 2008. 
33. Chen, T.; Peng, L.; Yin, X.; Jing, B.; Yang, J.; Cong, G.; Li, G. A Policy Category Analysis Model for Tourism Promotion in China During the COVID-19 Pandemic Based on Data Mining and Binary Regression. Risk Manag. Healthc. Policy 2020, 13, 3211-3233. [CrossRef]

34. Chen, T.; Peng, L.; Yang, J.; Cong, G. Analysis of User Needs on Downloading Behavior of English Vocabulary APPs Based on Data Mining for Online Comments. Mathematics 2021, 9, 1341. [CrossRef]

35. Adnerson, R.M.; May, R.M. Infectious diseases of humans: Dynamics and control. Science 1991, 254, 591-592.

36. Wallinga, J.; Lipsitch, M. How generation intervals shape the relationship between growth rates and reproductive numbers. Proc. Biol. Sci. 2007, 274, 599-604. [CrossRef] [PubMed]

37. Goh, K.I.; Kahng, B.; Kim, D. Universal behavior of load distribution in scale-free networks. Phys. Rev. Lett. 2001, 87, 278701. [CrossRef]

38. Chen, T.; Rong, J.; Peng, L.; Yang, J.; Cong, G.; Fang, J. Analysis of Social Effects on Employment Promotion Policies for College Graduates Based on Data Mining for Online Use Review in China during the COVID-19 Pandemic. Healthcare 2021, 9, 846. [CrossRef] [PubMed] 